\title{
TIME-MULTIPATCH DISCONTINUOUS GALERKIN SPACE-TIME ISOGEOMETRIC ANALYSIS OF PARABOLIC EVOLUTION PROBLEMS*
}

\author{
CHRISTOPH HOFER ${ }^{\dagger}$, ULRICH LANGER $^{\ddagger}$, MARTIN NEUMÜLLER ${ }^{\ddagger}$, AND IOANNIS TOULOPOULOS $§$
}

\begin{abstract}
In this paper, we present a new time-multipatch discontinuous Galerkin Isogeometric Analysis (IgA) technology for solving parabolic initial-boundary problems in space and time simultaneously. We prove coercivity of the IgA variational problem with respect to a suitably chosen norm that together with boundedness, consistency, and approximation results yields a priori discretization error estimates in this norm. Furthermore, we provide efficient parallel generation and parallel multigrid solution technologies. Finally, we present first numerical results on massively parallel computers.
\end{abstract}

Key words. parabolic initial-boundary value problems, space-time isogeometric analysis, time discontinuous Galerkin methods, space-time multigrid solvers

AMS subject classifications. 35K20, 65M12, 65M15, 65M55

1. Introduction. Fully discrete schemes for parabolic initial-boundary value problems (IBVP) are usually derived by discretizing either first in space by means of some spatial discretization method like the finite element method and then in time by some time-stepping method or vice versa. The former methods are called vertical methods of lines [55], the latter horizontal methods of lines or Rothe's methods [32]. Time-stepping methods are sequential in time. To overcome this curse of sequentiality on massively parallel computers, new methods must be developed for parallelizing in the time dimension. Time parallel methods have a long and exciting history that can be found in the very nice paper [19] on 50 Years of Time Parallel Time Integration. Space-time finite element methods for parabolic and hyperbolic partial differential equations (PDEs) go back to the 80s and 90s of the last century $[3,4,21,26,27,60]$ and have enjoyed a revival during the last couple of years due to the availability of massively parallel computers with thousands or hundred thousands of cores; see, e.g., $[1,2,5,6,37,38,40,41,46,47,48,56,57,58,59]$ for some resent mathematical papers related to parabolic problems. Moreover, there are several recent papers on the efficient use of various space-time methods for solving exciting engineering problems involving moving computational spatial domains and/or interfaces; see, e.g., [8, 28, 29, 50, 51, 52, 53, 54] and the references therein.

In [34], we were inspired by looking at the time variable $t$ in a parabolic problem as just another variable, say, $x_{d+1}$ if $x_{1}, \ldots, x_{d}$ are the spatial variables, and at the time derivative as a strong convection in the direction $x_{d+1}$ that can numerically be treated in a stable way by special discretization techniques known from convection-dominated elliptic convection-diffusion problems; see, e.g., [49]. The most popular stabilizing method is the Streamline-Upwind Petrov-Galerkin (SUPG) method introduced in [13]. We have used timeupwind test functions to construct stable single-patch space-time Isogeometric Analysis (IgA) schemes where the discrete bilinear form is coercive (elliptic) on the IgA space with respect to a suitably chosen mesh-dependent energy norm. This coercivity (ellipticity) property together with a corresponding boundedness property and consistency as well as approximation results

*Received May 9, 2017. Accepted April 6, 2018. Published online on June 12, 2018. Recommended by Susanne C. Brenner.

${ }^{\dagger}$ Doctoral Program “Computational Mathematics”, Johannes Kepler University, Altenbergerstr. 69, A-4040 Linz, Austria (christoph.hofer@dk-compmath. jku.at).

${ }^{\ddagger}$ Institute of Computational Mathematics, Johannes Kepler University, Altenbergerstr. 69, A-4040 Linz, Austria ( $\{$ ulanger, neumueller $\}$ @numa.uni-linz.ac.at).

$\S$ Johann Radon Institute for Computational and Applied Mathematics, Austrian Academy of Sciences, Altenbergerstr. 69, A-4040 Linz, Austria (ioannis.toulopoulos@ricam. oeaw.ac.at). 
for the IgA spaces yields the corresponding a priori discretization error estimates. A posteriori error estimates that can be used for space-time adaptivity are derived in [33].

IgA was introduced in [25] as a new discretization methodology for PDE-based models. The core idea of $\operatorname{IgA}$ is to use the same smooth and high-order superior finite-dimensional B-spline or NURBS spaces for parametrizing the computational domain and for approximating the solution of the PDE model under consideration. IgA approaches have successfully been applied to the solution of a wide range of linear and nonlinear problems. Their benefits have been highlighted in many publications; see, e.g., the monograph [14], the survey paper [10], and the references therein. Although results related to the approximation properties of Bsplines and their use for discretizing PDEs have been given before (see, e.g., [45] and [24]), the theoretical frame involving the parametrization mappings has been started in [7], where the authors studied the approximation properties of B-splines (NURBS) in bent Sobolev spaces. In particular, they showed that the mapped B-splines have the same approximation order in terms of the mesh size $h$ as the piecewise polynomials of the same degree $p$; see also $[9,10,11]$ for the generalization of these approximation results.

In this paper, we generalize the results of [34] from the single-patch to time-multipatch discontinuous Galerkin (dG) space-time IgA schemes. As in [34], we consider the linear parabolic IBVP: find $u: \bar{Q} \rightarrow \mathbb{R}$ such that

$$
\partial_{t} u-\Delta u=f \quad \text { in } Q, \quad u=0 \quad \text { on } \Sigma, \quad \text { and } \quad u=u_{0} \quad \text { on } \bar{\Sigma}_{0},
$$

as a typical parabolic model problem posed in the space-time cylinder $\bar{Q}=\bar{\Omega} \times[0, T]$ $=Q \cup \Sigma \cup \bar{\Sigma}_{0} \cup \bar{\Sigma}_{T}$, where $\partial_{t}$ denotes the partial time derivative, $\Delta$ is the Laplace operator, $f$ is a given source function, $u_{0}$ are the given initial data, $T$ is the final time, $Q=\Omega \times(0, T)$, $\Sigma=\partial \Omega \times(0, T), \Sigma_{0}:=\Omega \times\{0\}, \Sigma_{T}:=\Omega \times\{T\}$, and $\Omega \subset \mathbb{R}^{d}(d=1,2,3)$ denotes the spatial computational domain with the boundary $\partial \Omega$. The spatial domain $\Omega$ is supposed to have a singlepatch NURBS representation as it is used in CAD [44]. More precisely, the space-time cylinder $\bar{Q}=\bigcup_{n=1}^{N} \bar{Q}_{n}$ is composed of $N$ subcylinders (patches or time slices) $Q_{n}=\Omega \times\left(t_{n-1}, t_{n}\right), n=1, \ldots, N$, where $0=t_{0}<t_{1}<\ldots<t_{N}=T$ is some subdivision of the time interval $[0, T]$; see also Figure 2.1 for an illustration. The time faces between the time patches are denoted by $\bar{\Sigma}_{n}=\bar{Q}_{n+1} \cap \bar{Q}_{n}=\bar{\Omega} \times\left\{t_{n}\right\}$, where $\Sigma_{N}=\Sigma_{T}$. Every space-time patch $Q_{n}=\boldsymbol{\Phi}_{n}(\widehat{Q})$ in the physical domain $Q$ can be represented as the image of the parameter domain $\widehat{Q}=(0,1)^{d+1}$ by means of a sufficiently regular IgA (B-spline, NURBS, etc.) map $\boldsymbol{\Phi}_{n}: \widehat{Q} \rightarrow Q_{n}$ that can easily be constructed from the spatial IgA map from $\widehat{\Omega}=(0,1)^{d}$ to $\Omega$. In particular, each $Q_{n}$ can have its own mesh defined according to the characteristics of the problem. Therefore, the $\operatorname{IgA}$ spaces $V_{h 0}$, which we are going to use, are smooth in each time patch $Q_{n}$ but discontinuous across the time faces $\Sigma_{n}$. For stabilizing the time discretization, the method incorporates ideas of streamline diffusion methodology. The continuity of the patch-wise defined approximate solutions is ensured by introducing simple "upwind" jump terms across the interfaces. The jump terms do not include normal fluxes. This simplifies the error analysis. Moreover, the whole method can easily be implemented on a parallel platform. We develop a thorough theoretical study of the method. After defining the appropriate discontinuous B-spline spaces and the related discrete norm, we prove that the constructed discrete bilinear form is coercive (elliptic) with respect to this norm. This property ensures uniqueness and existence of the $\operatorname{IgA}$ solution. Based on this ellipticity result, a related boundedness result, and the consistency of the discrete bilinear form, we can easily estimate the discretization error by the best approximation error with respect to the discrete norm. With the help of the approximation results from $[10,11]$, we derive discretization error estimates taking into account that the exact solution can exhibit anisotropic regularity behavior, i.e., different regularity properties with respect to time and space directions. 


\section{ETNA}

Kent State University and

Johann Radon Institute (RICAM)

Finally, we have to solve one huge linear system $\mathbf{L}_{h} \mathbf{u}_{h}=\mathbf{f}_{h}$ of $\operatorname{IgA}$ equations defining all the control points in space and time all at once. The fast generation and the fast solution of this system is an issue. In our case, we can benefit from the special time-multipatch dG structure of the discretization that leads to a block-bidiagonal system matrix $\mathbf{L}_{h}=\operatorname{blockbidiag}\left(-\mathbf{B}_{i}, \mathbf{A}_{i}\right)$, where the block-diagonal matrices $\mathbf{A}_{i}, i=1, \ldots, N$, and the block-subdiagonal matrices $\mathbf{B}_{i}$, $i=2, \ldots, N$, have tensor-product representations. These properties lead to a fast generation of the matrix $\mathbf{L}_{h}$. The block-bidiagonal structure of the system matrix $\mathbf{L}_{h}$ enables us to solve the system sequentially from one space-time patch to the next space-time patch similar to a time-stepping scheme. However, we want to overcome this curse of sequentiality since we want to use the power of massively parallel computers with hundred or thousands of cores to solve this system efficiently. Similar to [20], we propose a space-time multigrid method that solves the complete system $\mathbf{L}_{h} \mathbf{u}_{h}=\mathbf{f}_{h}$ in parallel. In fact, we use the space-time multigrid method as preconditioner in a GMRES solver. The numerical results presented in the paper confirm not only our convergence rate estimates but also show the efficiency of the generation and solver technology proposed in the paper. The first numerical results for the lowest-order splines can be found in our proceedings paper [35], where we consider the simplified case of the same smoothness of the solution in space and time.

The remainder of the paper is organized as follows. In Section 2, besides introducing some notation and preliminaries, the stable time-multipatch dG space-time IgA scheme is derived. Section 3 provides a complete a priori discretization error analysis, whereas Section 4 gives the matrix representation of our time-multipatch dG space-time IgA scheme and describes the parallel space-time multigrid preconditioned GMRES solver. Finally, we present and discuss the first numerical results in Section 5 and draw some conclusions in Section 6.

\section{The model problem and its stable space-time IgA discretization.}

2.1. Preliminaries. Let $\Omega$ be a bounded Lipschitz domain in $\mathbb{R}^{d}, d=1,2$, or 3 , with the boundary $\Gamma=\partial \Omega$. For any multi-index $\boldsymbol{\alpha}=\left(\alpha_{1}, \ldots, \alpha_{d}\right)$ of non-negative integers $\alpha_{1}, \ldots, \alpha_{d}$, we define the differential operator $\partial_{x}^{\boldsymbol{\alpha}}=\partial_{x_{1}}^{\alpha_{1}} \ldots \partial_{x_{d}}^{\alpha_{d}}$, with $\partial_{x_{j}}=\partial / \partial x_{j}$, for $j=1, \ldots, d$. As usual, $L_{2}(\Omega)$ denotes the Lebesgue space of all Lebesgue measurable and square-integrable functions endowed with the norm $\|v\|_{L_{2}(\Omega)}=\left(\int_{\Omega}|v(x)|^{2} d x\right)^{\frac{1}{2}}$, and $L_{\infty}(\Omega)$ denotes the space of functions that are essentially bounded. For a non-negative integer $\ell$, we define the standard Sobolev space

$$
H^{\ell}(\Omega)=\left\{v \in L_{2}(\Omega): \partial_{x}^{\boldsymbol{\alpha}} v \in L_{2}(\Omega) \text { for all }|\boldsymbol{\alpha}|=\sum_{j=1}^{d} \alpha_{j} \leq \ell\right\}
$$

endowed with the norm

$$
\|v\|_{H^{\ell}(\Omega)}=\left(\sum_{0 \leq|\boldsymbol{\alpha}| \leq \ell}\left\|\partial_{x}^{\boldsymbol{\alpha}} v\right\|_{L_{2}(\Omega)}^{2}\right)^{\frac{1}{2}}
$$

whereas the trace space of $H^{1}(\Omega)$ is denoted by $H^{\frac{1}{2}}(\Gamma)$. Further, we introduce the subspace $H_{0}^{1}(\Omega)=\left\{v \in H^{1}(\Omega): v=0\right.$ on $\left.\Gamma\right\}$ of all functions $v$ from $H^{1}(\Omega)$ with zero traces on $\Gamma$. Let $J=(0, T)$ be the time interval with some final time $T>0$. For later use, we define the space-time cylinder $Q=\Omega \times J$ and its boundary parts $\Sigma=\partial \Omega \times J, \Sigma_{T}=\Omega \times\{T\}$, and $\Sigma_{0}=\Omega \times\{0\}$ such that $\partial Q=\Sigma \cup \bar{\Sigma}_{0} \cup \bar{\Sigma}_{T}$. Analogously to the definition of $\partial_{x}^{\boldsymbol{\alpha}}$, we now define the spatial gradient $\nabla_{x} v=\left(\partial_{x_{1}} v, \ldots, \partial_{x_{d}} v\right)$. Let $\ell$ and $m$ be non-negative integers. 
For functions defined in the space-time cylinder $Q$, we introduce the Sobolev spaces

$$
\begin{aligned}
H^{\ell, m}(Q)=\left\{v \in L_{2}(Q):\right. & \partial_{x}^{\boldsymbol{\alpha}} v \in L_{2}(Q) \text { for } 0 \leq|\boldsymbol{\alpha}| \leq \ell, \\
& \text { and } \left.\partial_{t}^{i} v \in L_{2}(Q), i=0,1, \ldots, m\right\},
\end{aligned}
$$

where $\partial_{t}=\partial / \partial t$ and, in particular, with the subspaces

$$
\begin{aligned}
& H_{0}^{1,0}(Q)=\left\{v \in L_{2}(Q): \nabla_{x} v \in\left[L_{2}(Q)\right]^{d}, v=0 \text { on } \Sigma\right\} \quad \text { and } \\
& H_{0, \overline{0}}^{1,1}(Q)=\left\{v \in L_{2}(Q): \nabla_{x} v \in\left[L_{2}(Q)\right]^{d}, \partial_{t} v \in L_{2}(Q), v=0 \text { on } \Sigma, v=0 \text { on } \Sigma_{T}\right\} .
\end{aligned}
$$

We equip the above spaces with the following norms and seminorms

and

$$
\|v\|_{H^{\ell, m}(Q)}=\left(\sum_{|\alpha| \leq \ell}\left\|\partial_{x}^{\left(\alpha_{1}, \ldots, \alpha_{d}\right)} v\right\|_{L_{2}(Q)}^{2}+\sum_{i=0}^{m}\left\|\partial_{t}^{i} v\right\|_{L_{2}(Q)}^{2}\right)^{\frac{1}{2}}
$$

$$
|v|_{H^{\ell, m}(Q)}=\left(\sum_{|\boldsymbol{\alpha}|=\ell}\left\|\partial_{x}^{\left(\alpha_{1}, \ldots, \alpha_{d}\right)} v\right\|_{L_{2}(Q)}^{2}+\left\|\partial_{t}^{m} v\right\|_{L_{2}(Q)}^{2}\right)^{\frac{1}{2}},
$$

respectively. Furthermore, we recall Cauchy's and Young's inequalities

$$
\left|\int_{\Omega} u v d x\right| \leq\|u\|_{L_{2}(\Omega)}\|v\|_{L_{2}(\Omega)} \quad \text { and } \quad\left|\int_{\Omega} u v d x\right| \leq \frac{\epsilon}{2}\|u\|_{L_{2}(\Omega)}^{2}+\frac{1}{2 \epsilon}\|v\|_{L_{2}(\Omega)}^{2},
$$

which hold for all functions $u$ and $v$ from $L_{2}(\Omega)$ and for any fixed $\epsilon \in(0, \infty)$. We also recall Friedrichs' inequality, which we later need in the form

$$
\|v\|_{L_{2}(Q)} \leq C_{\Omega}\left\|\nabla_{x} v\right\|_{L_{2}(Q)},
$$

which holds for all $v \in H^{1}(Q)$ with vanishing trace on $\Sigma$; see, e.g., [12] for a proof of Friedrichs' inequality.

In what follows, positive constants $c$ and $C$ appearing in inequalities are generic constants which do not depend on the mesh-size $h$. In many cases, we will indicate what the constants may depend on for an easier understanding of the proofs. Frequently, we will write $a \sim b$ meaning that $c a \leq b \leq C a$ with generic positive constants $c$ and $C$.

2.2. The parabolic model problem. Using the standard procedure and integration by parts with respect to both $x$ and $t$, we can easily derive the following space-time variational formulation of (1.1): find $u \in H_{0}^{1,0}(Q)$ such that

$$
a(u, v)=l(v) \quad \text { for all } v \in H_{0, \overline{0}}^{1,1}(Q),
$$

with the bilinear form

$$
a(u, v)=-\int_{Q} u(x, t) \partial_{t} v(x, t) d x d t+\int_{Q} \nabla_{x} u(x, t) \cdot \nabla_{x} v(x, t) d x d t
$$

and the linear form

$$
l(v)=\int_{Q} f(x, t) v(x, t) d x d t+\int_{\Omega} u_{0}(x) v(x, 0) d x,
$$

where the source $f \in L_{2}(Q)$ and the initial conditions $u_{0} \in L_{2}(\Omega)$ are given. 


\section{ETNA}

Kent State University and

Johann Radon Institute (RICAM)

For simplicity, we only consider homogeneous Dirichlet boundary conditions on $\Sigma$. However, the analysis presented in our paper can easily be generalized to other classes of boundary conditions. The space-time variational formulation (2.3) has a unique solution; see, e.g., [30, 31]. In these monographs, besides existence and uniqueness results, one can also find useful a priori estimates and regularity results.

ASSUMPTION 2.1. We assume that the solution $u$ of (2.3) belongs to the space $V=H_{0}^{1,0}(Q) \cap H^{\ell, m}(Q)$ with some $\ell \geq 2$ and $m \geq 1$.

2.3. B-spline spaces and patch parametrizations. In this section, we briefly present the B-spline spaces and the B-spline parametrizations for the physical space-time patches (subdomains). We refer to $[14,15,45]$ for a more detailed introduction to B-splines.

To describe more clearly the basic material, we start with the B-spline spaces for the univariate case. Let the integer $p_{1}$ denote the $\mathrm{B}$-spline degree and the integer $n_{1}$ denote the number of basis functions. We now consider a partition $\mathcal{Z}=\left\{0=z_{1}, z_{2}, \ldots, z_{M}=1\right\}$ of the interval $[0,1]$ with the subintervals $\left[z_{j}, z_{j+1}\right]$, where $j=1, \ldots, M-1$. Based on $\mathcal{Z}$, we introduce a knot-vector $\Xi=\left\{0=\xi_{1} \leq \xi_{2} \leq \ldots \leq \xi_{n_{1}+p_{1}+1}=1\right\}$ and the associated vector of the knot repetitions $\mathcal{M}=\left\{m_{1}, \ldots, m_{M}\right\}$. This means,

$$
\Xi=\{\underbrace{0=\xi_{1}, \ldots, \xi_{m_{1}}}_{=z_{1}}, \underbrace{\xi_{m_{1}+1}=\ldots=\xi_{m_{1}+m_{2}}}_{=z_{2}}, \ldots, \underbrace{\xi_{n_{1}+p_{1}+1-m_{M}}, \ldots, \xi_{n_{1}+p_{1}+1}=1}_{=z_{M}}\} .
$$

We assume that $m_{1}=m_{M}=p_{1}+1$ and $m_{j} \leq p_{1}$ for all internal knots. Such a knot-vector $\Xi$ is also called open knot-vector. The B-spline basis functions are defined by the Cox-de Boor recursion formula

$$
B_{i, j}=\frac{x-\xi_{i}}{\xi_{i+j}-\xi_{i}} B_{i, j-1}(x)+\frac{\xi_{i+j+1}-x}{\xi_{i+j+1}-\xi_{i+1}} B_{i+1, j-1}(x),
$$

where $j$ is running from 1 to $p_{1}$ and $B_{i, 0}(x)=1$ if $\xi_{i} \leq x \leq \xi_{i+1}$ and 0 otherwise; see, e.g., $[14,15]$.

The multivariate B-spline spaces can easily be derived through tensor-product procedures of the univariate spaces. Let us consider the unit cube $\widehat{Q}=(0,1)^{d+1} \subset \mathbb{R}^{d+1}$, which we will refer to as the parametric domain. Following the same steps as in the univariate case, we consider integers $p_{k}$ and $n_{k}$ that indicate the given B-spline degree and the number of basis functions of the B-spline space in the $x_{k}$-direction, respectively, with $k=1, \ldots, d+1$. We introduce the corresponding open-knot vectors $\Xi^{k}=\left\{0=\xi_{1}^{k} \leq \xi_{2}^{k} \leq \ldots \leq \xi_{n_{k}+p_{k}+1}^{k}=1\right\}$, the vectors $\mathcal{Z}^{k}$, and the vectors $\mathcal{M}^{k}$. We associate with each knot vector $\Xi^{k}$ the space $\hat{\mathbb{B}}_{\Xi^{k}}$ of B-spline basis functions of degree $p_{k}$, where $k=1, \ldots, d+1$. On the parametric domain $\hat{Q}$, we define the tensor-product $\mathrm{B}$-spline space $\hat{\mathbb{B}}_{\Xi^{d+1}}=\bigotimes_{k=1}^{d+1} \hat{\mathbb{B}}_{\Xi^{k}}$, where $\Xi^{d+1}=\left(\Xi^{1}, \ldots, \Xi^{k}, \ldots, \Xi^{d+1}\right)$.

In practice, it is usually more convenient to describe the computational domain as a union of subdomains (patches) and to develop a multi-patch IgA approach. In this paper, we describe the space-time cylinder $Q$ as a union of non-overlapping time patches $Q_{1}, Q_{2}, \ldots, Q_{N}$. We consider a partition $0=t_{0}<t_{1}<\ldots<t_{N}=T$ of the time interval $[0, T]$ and denote the subintervals by $J_{n}=\left(t_{n-1}, t_{n}\right)$. We now define the time patches $Q_{n}=\Omega \times J_{n}$ and the faces $\Sigma_{n}=\bar{Q}_{n+1} \cap \bar{Q}_{n}=\Omega \times\left\{t_{n}\right\}$ between the time patches, where we identify $\Sigma_{T}$ and $\Sigma_{N}$. In that way, we have the decomposition $\bar{Q}=\bigcup_{n=1}^{N} \bar{Q}_{n}$. A schematic illustration is presented in Figure 2.1.

We now proceed with the definition of the B-spline spaces in every $Q_{n}$ as well as the corresponding parametrizations. For simplicity, let us assume that the B-spline degree is the same for all directions and for all time patches $Q_{n}$, i.e., $p_{k, n}=p$ for $k=1, \ldots, d+1$, and let 


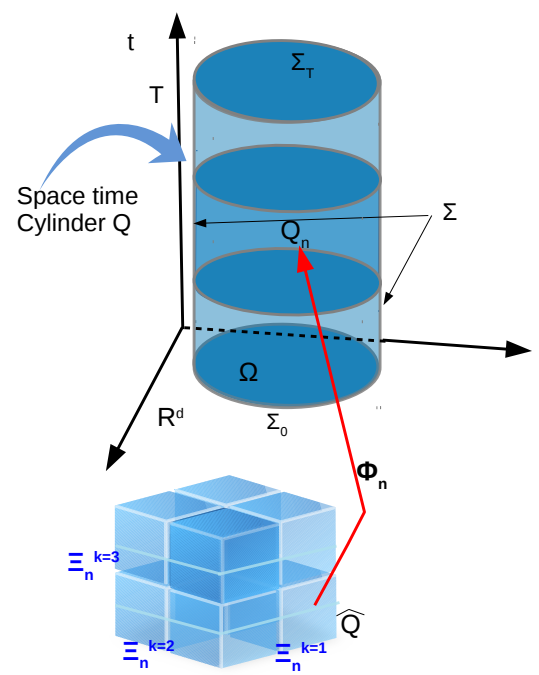

(a)

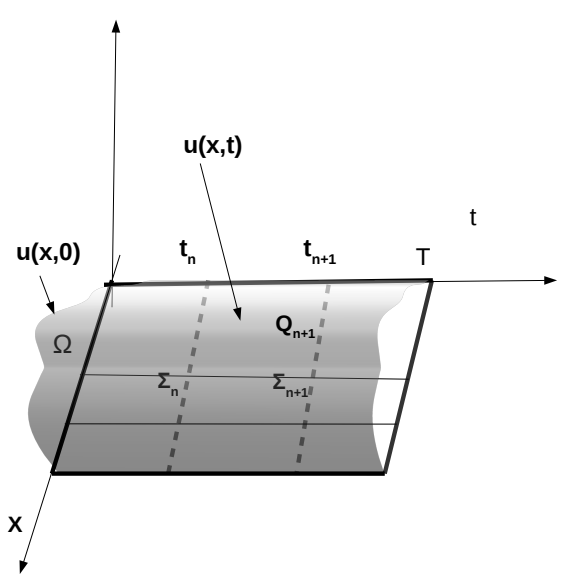

(b)

FIG. 2.1. (a) The decomposition of the space-type cylinder $Q$ into space-time patches $Q_{n}$ together with the mesh in the parametric domain $\widehat{Q}$ produced by the knot-vectors $\Xi_{n}^{k}$. (b) The space-time patches $Q_{n}$ with their interfaces and the graph of $u$ for the case of $Q \subset \mathbb{R}^{2}$.

the integer $n_{k, n}$ denote the number of basis functions of the B-spline space in $x_{k}$-direction. In the following, we simply write $n_{k}$ for $n_{k, n}$, but we allow different $n_{k}$ in different time patches $Q_{n}$, with $n=1, \ldots, N$. For every $Q_{n}$, we introduce the $(d+1)$-dimensional vector of knot-vectors $\Xi_{n}^{d+1}=\left(\Xi_{n}^{1}, \ldots, \Xi_{n}^{k}, \ldots, \Xi_{n}^{d+1}\right)$, with the particular components given by $\Xi_{n}^{k}=\left\{0=\xi_{1}^{k} \leq \xi_{2}^{k} \leq \ldots \leq \xi_{n_{k}+p+1}^{k}=1\right\}$. For all the internal knots, we again assume that $m_{j}^{k} \leq p$, with $m_{j}^{k}$ being the associated multiplicities. The components $\Xi_{n}^{k}$ of $\Xi_{n}^{d+1}$ induce a mesh $T_{\hat{h}_{n}}^{n}(\widehat{Q})=\left\{\hat{E}_{m}\right\}_{m=1}^{M_{n}}$ in $\widehat{Q}$, where $\hat{E}_{m}$ are the micro elements and $\hat{h}_{n}$ is the mesh size, which is defined as follows: given a micro element $\hat{E}_{m} \in T_{\hat{h}_{n}}^{n}(\widehat{Q})$, we set $\hat{h}_{\hat{E}_{m}}=\operatorname{diam}\left(\hat{E}_{m}\right)$, and we define $\hat{h}_{n}=\max \left\{\hat{h}_{\hat{E}_{m}}\right\}$. We set $\hat{h}=\max _{n=1, \ldots, N}\left\{\hat{h}_{n}\right\}$. We refer the reader to [14] for more information about the meaning of the knot vectors in CAD and IgA.

Given the knot vector $\Xi_{n}^{k}$ in every direction $k=1, \ldots, d+1$, we construct the associated univariate B-spline basis functions $\left\{\hat{B}_{1, k}^{n}\left(\hat{x}_{k}\right), \ldots, \hat{B}_{n_{k}, k}^{n}\left(\hat{x}_{k}\right)\right\}$ using the Cox-de Boor recursion formula (2.4); see also $[14,15]$ for more details. Accordingly, on the mesh $T_{\hat{h}_{n}}^{n}(\widehat{Q})$, the basis functions of the multivariate B-spline space $\hat{\mathbb{B}}_{\Xi_{n}^{d+1}}$ are defined by the tensor-product of the corresponding univariate $\mathrm{B}$-spline basis functions spanning the spaces $\hat{\mathbb{B}}_{\Xi_{n}^{k}}$, i.e.,

$$
\hat{\mathbb{B}}_{\Xi_{n}^{d+1}}=\bigotimes_{k=1}^{d+1} \hat{\mathbb{B}}_{\Xi_{n}^{k}}=\operatorname{span}\left\{\hat{B}_{j}^{n}(\hat{x})\right\}_{j=1}^{n_{B}=n_{1} \cdot \ldots \cdot n_{k} \cdot \ldots \cdot n_{d+1}}
$$

where each $\hat{B}_{j}^{n}(\hat{x})$ has the form

$$
\hat{B}_{j}^{n}(\hat{x})=\hat{B}_{j_{1}}^{n}\left(\hat{x}_{1}\right) \cdot \ldots \cdot \hat{B}_{j_{k}}^{n}\left(\hat{x}_{k}\right) \cdot \ldots \cdot \hat{B}_{j_{d}}^{n}\left(\hat{x}_{d}\right)
$$


According to the IgA approach, every time patch $Q_{n}$ is the image of the parameter domain by means of a parametrization mapping $\boldsymbol{\Phi}_{n}$ defined by the control points and the corresponding B-spline basis functions. More precisely, we assume that we are given the control points $\mathbf{C}_{j}^{n}$ related to $Q_{n}$, and we parametrize each space-time patch $Q_{n}$ by

$$
\boldsymbol{\Phi}_{n}: \widehat{Q} \rightarrow Q_{n}=\boldsymbol{\Phi}_{n}(\widehat{Q}), \quad x=\boldsymbol{\Phi}_{n}(\hat{x})=\sum_{j=1}^{n_{B}} \mathbf{C}_{j}^{n} \hat{B}_{j}^{n}(\hat{x}),
$$

where $\hat{x}=\boldsymbol{\Phi}_{n}^{-1}(x), n=1, \ldots, N$; cf. [14].

For every $Q_{n}$, we construct a mesh $T_{h_{Q_{n}}^{n}}^{n}\left(Q_{n}\right)=\left\{E_{m}\right\}_{m=1}^{M_{n}}$, where the elements $E_{m}$ are the images of $\hat{E}_{m} \in T_{\hat{h}_{n}}^{n}(\widehat{Q})$ under $\boldsymbol{\Phi}_{n}$, i.e., $E_{m}=\boldsymbol{\Phi}_{n}\left(\hat{E}_{m}\right)$. Also, for each $E \in T_{h_{Q_{n}}}^{n}\left(Q_{n}\right)$, we denote its support extension by $\widetilde{E}$, where the support extension is defined to be the interior of the set formed by the union of the supports of all B-spline functions whose supports intersects $E$. Accordingly to the parametric mesh, we let $h_{E_{m}}=\operatorname{diam}\left(E_{m}\right)$ and define $h_{Q_{n}}=\max \left\{h_{E_{m}}: E_{m} \in T_{h_{Q_{n}}}^{n}\left(Q_{n}\right)\right\}$, and the global physical mesh size is $h=\max h_{Q_{n}}$. For $n=1, \ldots, N$, we construct the $\mathrm{B}$-spline space $\mathbb{B}_{\Xi_{n}^{d+1}}$ on $Q_{n}$ by

$$
\mathbb{B}_{\boldsymbol{\Xi}_{n}^{d+1}}:=\operatorname{span}\left\{\left.B_{j}^{n}\right|_{Q_{n}}: B_{j}^{n}(x)=\hat{B}_{j}^{n} \circ \boldsymbol{\Phi}_{n}^{-1}(x), \text { for } j=1, \ldots, n_{B}\right\} .
$$

The global B-spline space $V_{h}$ with components on every $\mathbb{B}_{\Xi_{n}^{d}}$ is defined by

$$
V_{h}:=V_{h_{1}}^{1} \times \ldots \times V_{h_{N}}^{N}:=\mathbb{B}_{\boldsymbol{\Xi}_{1}^{d+1}} \times \ldots \times \mathbb{B}_{\boldsymbol{\Xi}_{N}^{d+1}} .
$$

ASSUMPTION 2.2. The meshes $T_{\hat{h}_{n}}^{n}(\widehat{Q})$ are uniform, i.e., for every $\hat{E} \in T_{\hat{h}_{n}}^{n}(\widehat{Q})$, there exist a number $\gamma_{n}>0$ such that $\gamma_{n} \leq \hat{h}_{n} / \rho_{\hat{E}}$, where $\rho_{\hat{E}}$ is the radius of the inscribed circle of $\hat{E}$.

REMARK 2.3. Since the parametrizations $\boldsymbol{\Phi}_{n}, n=1, \ldots, N$, are fixed, under Assumption 2.2, we have that $\hat{h}_{n} \sim h_{Q_{n}}$. Thus, below, we use $h_{n}$ for denoting any of the mesh sizes, parametric or physical. For simplicity, we assume that $h_{n} \leq 1$ for all $n=1, \ldots, N$.

The parametrization mappings $\boldsymbol{\Phi}_{n}, n=1, \ldots, N$, can be considered to be bi-Lipschitz homeomorphisms [10]. To simplify the analysis, we further consider the following regularity properties imposed on $\boldsymbol{\Phi}_{n}, n=1, \ldots, N$.

AsSUMPTION 2.4. Assume that every $\boldsymbol{\Phi}_{n}$ and $\boldsymbol{\Phi}_{n}^{-1}, n=1, \ldots, N$, are sufficiently smooth ( $C^{1}$ diffeomorphisms) and that there exist constants $0<c<C$ such that $c \leq\left|\operatorname{det} J_{\mathbf{\Phi}_{n}}\right| \leq C$, where $J_{\mathbf{\Phi}_{n}}$ is the Jacobian matrix of $\boldsymbol{\Phi}_{n}$, i.e., $J_{\mathbf{\Phi}_{n}}=\frac{\partial\left(\boldsymbol{\Phi}_{n, 1}, \ldots, \boldsymbol{\Phi}_{n, d+1}\right)}{\partial\left(\hat{x}_{1}, \ldots, \hat{x}_{d}, \hat{t}\right)}$.

Assumption 2.4 helps in simplifying the form of the constants which appear in the relations between the norms of the pull-back solution and the physically relevant solution.

COROLlaRY 2.5. Let Assumption 2.4 hold, and let $u \in H^{\ell, m}(Q)$ with $\ell \geq 2$ and $m \geq 1$. Then its pull-back $\widehat{u}=u \circ \boldsymbol{\Phi}_{n}$ is in $H^{1,1}(\widehat{Q})$, and there exist constants $c_{1}$ and $c_{2}$ depending only on $\boldsymbol{\Phi}_{n}$ and $\boldsymbol{\Phi}_{n}^{-1}$ and not on $u$ such that $c_{1}\|\widehat{u}\|_{H^{1,1}(\widehat{Q})} \leq\|u\|_{H^{1,1}\left(Q_{n}\right)} \leq c_{2}\|\widehat{u}\|_{H^{1,1}(\widehat{Q})}$.

To keep the notation simple, in what follows, we will use the superscript $n$ to denote the restrictions to $Q_{n}$, e.g., $u^{n}:=\left.u\right|_{Q_{n}}$.

We denote the global discontinuous B-spline space and the local continuous patch-wise B-spline spaces by

$$
V_{0 h}=\left\{v_{h} \in L_{2}(Q):\left.v_{h}\right|_{Q_{n}} \in \mathbb{B}_{\Xi_{n}^{d+1}}\left(Q_{n}\right), \text { for } n=1, \ldots, N, \text { and }\left.v_{h}\right|_{\Sigma}=0\right\}
$$

and

$$
V_{0 h}^{n}=\left\{v_{h} \in \mathbb{B}_{\Xi_{n}^{d+1}}\left(Q_{n}\right), \text { for } n=1, \ldots, N, \text { and }\left.v_{h}\right|_{\Sigma}=0\right\}
$$


respectively. Notice that $v_{h} \in V_{0 h}$ is discontinuous across $\Sigma_{n}$. We introduce the notations

$$
\begin{aligned}
v_{h,+}^{n} & =\lim _{\varepsilon \rightarrow 0^{+}} v_{h}\left(t_{n}+\varepsilon\right), & v_{h,-}^{n} & =\lim _{\varepsilon \rightarrow 0^{-}} v_{h}\left(t_{n}+\varepsilon\right), \\
\llbracket v_{h} \rrbracket^{n} & =v_{h,+}^{n}-v_{h,-}^{n}, & \llbracket v_{h} \rrbracket^{0} & =v_{h,+}^{0},
\end{aligned}
$$

where $\llbracket v_{h} \rrbracket^{n}$ denotes the jump of $v_{h}$ across $\Sigma_{n}$, for $n \geq 1$, and $\llbracket v_{h} \rrbracket^{0}=v_{h,+}^{0}$ denotes the trace of $v_{h}$ on $\Sigma_{0}$. For a smooth function $u$, we obviously have $\llbracket u \rrbracket^{n}=u_{+}^{n}-u_{-}^{n}=0$, for $n \geq 1$, and $\llbracket u \rrbracket^{0}=\left.u\right|_{\Sigma_{0}}$.

2.4. Stable multipatch space-time dG IgA discretization. Let us consider the spacetime patch $Q_{n}$, and let us denote the outer normal to $\partial Q$ by $\mathbf{n}=\left(n_{1}, \ldots, n_{d}, n_{d+1}\right)=\left(\mathbf{n}_{x}, n_{t}\right)$. For the time being, we assume that $u^{n-1}$ is known. Let $v_{h}^{n} \in V_{0 h}^{n}$ and $w_{h}^{n}=v_{h}^{n}+\theta_{n} h_{n} \partial_{t} v_{h}^{n}$ with some positive parameter $\theta_{n}$, which will be defined later. Note that $\left.w_{h}^{n}\right|_{\Sigma}=0$. Multiplying $\partial_{t} u-\Delta u=f$ by $w_{h}^{n}$, integrating over $Q_{n}$, and applying integration by parts, we arrive at the variational identity

$$
\begin{gathered}
\int_{Q_{n}}\left(\partial_{t} u\left(v_{h}^{n}+\theta_{n} h_{n} \partial_{t} v_{h}^{n}\right)+\nabla_{x} u \cdot \nabla_{x} v_{h}^{n}+\theta_{n} h_{n} \nabla_{x} u \cdot \nabla_{x} \partial_{t} v_{h}^{n}\right) d x d t \\
-\int_{\partial Q_{n}} n_{x} \cdot \nabla_{x} u\left(v_{h}^{n}+\theta_{n} h_{n} \partial_{t} v_{h}^{n}\right) d x+\int_{\Sigma_{n-1}} u_{+}^{n-1} v_{h,+}^{n-1} d x \\
=\int_{Q_{n}} f\left(v_{h}^{n}+\theta_{n} h_{n} \partial_{t} v_{h}^{n}\right) d x d t+\int_{\Sigma_{n-1}} u_{-}^{n-1} v_{h,+}^{n-1} d x
\end{gathered}
$$

for $n=1, \ldots, N$, where we used that $u_{-}^{n-1}=u_{+}^{n-1}=u^{n-1}$ on every $\Sigma_{n-1}$. Furthermore, using $\left.n_{x}\right|_{\Sigma_{n}}=0$ and $w_{h}=0$ on $\Sigma$, we have

$$
\begin{aligned}
a_{Q_{n}}\left(u, v_{h}\right):= & \int_{Q_{n}}\left(\partial_{t} u\left(v_{h}^{n}+\theta_{n} h_{n} \partial_{t} v_{h}^{n}\right)+\nabla_{x} u \cdot \nabla_{x} v_{h}^{n}+\theta_{n} h_{n} \nabla_{x} u \cdot \nabla_{x} \partial_{t} v_{h}^{n}\right) d x d t \\
& \quad+\int_{\Sigma_{n-1}} \llbracket u \rrbracket^{n-1} v_{h,+}^{n-1} d x \\
= & \int_{Q_{n}} f\left(v_{h}^{n}+\theta_{n} h_{n} \partial_{t} v_{h}^{n}\right) d x d t
\end{aligned}
$$

for all $n=2, \ldots, N$, and

$$
\begin{aligned}
a_{Q_{1}}\left(u, v_{h}\right):= & \int_{Q_{1}}\left(\partial_{t} u\left(v_{h}^{1}+\theta_{1} h_{1} \partial_{t} v_{h}^{1}\right)+\nabla_{x} u \cdot \nabla_{x} v_{h}^{1}+\theta_{1} h_{1} \nabla_{x} u \cdot \nabla_{x} \partial_{t} v_{h}^{1}\right) d x d t \\
& \quad+\int_{\Sigma_{0}} \llbracket u \rrbracket^{0} v_{h,+}^{0} d x \\
= & \int_{Q_{1}} f\left(v_{h}^{1}+\theta_{1} h_{1} \partial_{t} v_{h}^{1}\right) d x d t+\int_{\Sigma_{0}} u_{0} v_{h,+}^{0} d x .
\end{aligned}
$$

Summing over all $Q_{n}$, we conclude that

$$
a_{h}\left(u, v_{h}\right)=l_{h}\left(v_{h}\right), \quad \forall v_{h} \in V_{0 h},
$$

where

$$
a_{h}\left(u, v_{h}\right)=\sum_{n=1}^{N} a_{Q_{n}}\left(u, v_{h}\right)
$$


and

$$
l_{h}\left(v_{h}\right)=\sum_{n=1}^{N} \int_{Q_{n}} f\left(v_{h}^{n}+\theta_{n} h_{n} \partial_{t} v_{h}^{n}\right) d x d t+\int_{\Sigma_{0}} u_{0} v_{h,+}^{0} d x
$$

Now, the space-time dG IgA variational scheme for (1.1) can be formulated as follows: find $u_{h} \in V_{0 h}$ such that

$$
a_{h}\left(u_{h}, v_{h}\right)=l_{h}\left(v_{h}\right), \quad \forall v_{h} \in V_{0 h} .
$$

In the following lemma, we cite a few auxiliary results that will be used in the error analysis below. For the proofs, we refer to [7, 9, 16]; see also the discussion in [36].

LEMMA 2.6. Let $Q_{n}$ be a space-time patch, and let $v \in H^{1}\left(Q_{n}\right), v_{h} \in \mathbb{B}_{\Xi_{n}^{d+1}}$, and $E \in T_{h_{n}}^{n}\left(Q_{n}\right)$. Then there are positive constants $C_{t r}, C_{i n v, 0}$, and $C_{i n v, 1}$ depending on $\boldsymbol{\Phi}_{n}$ and the quasi-uniform properties of $T_{h_{n}}^{n}\left(Q_{n}\right)$ such that

$$
\begin{aligned}
\|v\|_{L_{2}(\partial E)}^{2} & \leq C_{t r} h_{n}^{-1}\left(\|v\|_{L_{2}(E)}+h_{n}|v|_{H^{1}(E)}\right)^{2} \\
\left\|v_{h}\right\|_{L_{2}(\partial E)}^{2} & \leq C_{i n v, 0} h_{n}^{-1}\left\|v_{h}\right\|_{L_{2}(E)}^{2} \\
\left\|\nabla v_{h}\right\|_{L_{2}(E)}^{2} & \leq, C_{i n v, 1} h_{n}^{-2}\left\|v_{h}\right\|_{L_{2}(E)}^{2}
\end{aligned}
$$

By the inequalities (2.10), we can easily infer the inverse inequalities

$$
\left\|\partial_{t} v_{h}\right\|_{L_{2}(E)}^{2} \leq C_{i n v, 1} h_{n}^{-2}\left\|v_{h}\right\|_{L_{2}(E)}^{2}
$$

and

$$
\left\|\partial_{t} \partial_{x_{i}} v_{h}\right\|_{L_{2}(E)}^{2} \leq C_{i n v, 1} h_{n}^{-2}\left\|\partial_{x_{i}} v_{h}\right\|_{L_{2}(E)}^{2} .
$$

Motivated by the definition of the bilinear form $a_{h}(\cdot, \cdot)$ in (2.9), we introduce the meshdependent dG norm

$$
\begin{aligned}
\|v\|_{d G}=( & \sum_{n=1}^{N}\left(\left\|\nabla_{x} v\right\|_{L_{2}\left(Q_{n}\right)}^{2}+\theta_{n} h_{n}\left\|\partial_{t} v\right\|_{L_{2}\left(Q_{n}\right)}^{2}+\frac{1}{2}\left\|\llbracket v \rrbracket^{n-1}\right\|_{L_{2}\left(\Sigma_{n-1}\right)}^{2}\right) \\
& \left.+\frac{1}{2}\|v\|_{L_{2}\left(\Sigma_{N}\right)}^{2}\right)^{\frac{1}{2}}
\end{aligned}
$$

in which we are going to estimate the discretization error.

LEMMA 2.7. The discrete bilinear form $a_{h}(\cdot, \cdot)$ defined in (2.9) is $V_{0 h}$-elliptic, i.e.,

$$
a_{h}\left(v_{h}, v_{h}\right) \geq C_{e}\left\|v_{h}\right\|_{d G}^{2}, \quad \text { for } v_{h} \in V_{0 h},
$$

where $C_{e}=0.5$ for $0<\theta_{n} \leq C_{i n v, 0}^{-2}$.

Proof. Using Green's formula $\int_{Q_{n}}\left(\partial_{t} v_{h} v_{h}+v_{h} \partial_{t} v_{h}\right) d x d t=\int_{\partial Q_{n}} n_{t} v_{h}^{2} d s$, we obtain the identity

$$
\int_{Q_{n}} \partial_{t} v_{h} v_{h} d x d t=\frac{1}{2} \int_{Q_{n}} \partial_{t} v_{h}^{2} d x d t=\frac{1}{2} \int_{\Sigma_{n}}\left(v_{h,-}^{n}\right)^{2} d x-\frac{1}{2} \int_{\Sigma_{n-1}}\left(v_{h,+}^{n-1}\right)^{2} d x
$$


The definition of $a_{Q_{n}}(\cdot, \cdot)$ and the identity (2.12) yield

$$
\begin{aligned}
a_{Q_{n}}\left(v_{h}, v_{h}\right)= & \int_{Q_{n}}\left(\frac{1}{2} \partial_{t} v_{h}^{2}+\theta_{n} h_{n}\left(\partial_{t} v_{h}\right)^{2}+\left|\nabla_{x} v_{h}\right|^{2}+\frac{\theta_{n} h_{n}}{2} \partial_{t}\left|\nabla_{x} v_{h}\right|^{2}\right) d x d t \\
& +\int_{\Sigma_{n-1}} \llbracket v_{h} \rrbracket^{n-1} v_{h,+}^{n-1} d x \\
= & \int_{Q_{n}}\left(\theta_{n} h_{n}\left(\partial_{t} v_{h}\right)^{2}+\left|\nabla_{x} v_{h}\right|^{2}\right) d x d t+\int_{\partial Q_{n}} \frac{\theta_{n} h_{n}}{2}\left|\nabla_{x} v_{h}\right|^{2} n_{t} d s \\
& \quad+\int_{\Sigma_{n-1}}\left(\left(v_{h,+}^{n-1}\right)^{2}-v_{h,-}^{n-1} v_{h,+}^{n-1}-\frac{1}{2}\left(v_{h,+}^{n-1}\right)^{2}\right) d x+\frac{1}{2} \int_{\Sigma_{n}}\left(v_{h,-}^{n}\right)^{2} d x \\
= & \theta_{n} h_{n}\left\|\partial_{t} v_{h}\right\|_{L_{2}\left(Q_{n}\right)}^{2}+\left\|\nabla_{x} v_{h}\right\|_{L_{2}\left(Q_{n}\right)}^{2} \\
& \quad+\frac{\theta_{n} h_{n}}{2}\left(\left\|\nabla_{x} v_{h}\right\|_{L_{2}\left(\Sigma_{n}\right)}^{2}-\left\|\nabla_{x} v_{h}\right\|_{L_{2}\left(\Sigma_{n-1}\right)}^{2}\right) \\
& \quad+\int_{\Sigma_{n-1}}\left(\left(v_{h,+}^{n-1}\right)^{2}-v_{h,-}^{n-1} v_{h,+}^{n-1}-\frac{1}{2}\left(v_{h,+}^{n-1}\right)^{2}\right) d x+\frac{1}{2} \int_{\Sigma_{n}}\left(v_{h,-}^{n}\right)^{2} d x \\
\geq & \left.\theta_{n} h_{n}\left\|\partial_{t} v_{h}\right\|_{L_{2}\left(Q_{n}\right)}^{2}+\left\|\nabla_{x} v_{h}\right\|_{L_{2}\left(Q_{n}\right)}^{2}-\frac{\theta_{n} h_{n}}{2}\left\|\nabla_{x} v_{h}\right\|_{L_{2}\left(\Sigma_{n-1}\right)}^{2}\right) \\
& \quad+\int_{\Sigma_{n-1}}\left(\frac{1}{2}\left(v_{h,+}^{n-1}\right)^{2}-v_{h,-}^{n-1} v_{h,+}^{n-1}\right) d x+\frac{1}{2} \int_{\Sigma_{n}}\left(v_{h,-}^{n}\right)^{2} d x \\
\geq & \theta_{n} h_{n}\left\|\partial_{t} v_{h}\right\|_{L_{2}\left(Q_{n}\right)}^{2}+\left(1-\frac{\left.\theta_{n} C_{i n v, 0}^{2}\right)\left\|\nabla_{x} v_{h}\right\|_{L_{2}\left(Q_{n}\right)}^{2}}{2}\right. \\
& +\int_{\Sigma_{n-1}}\left(\frac{1}{2}\left(v_{h,+}^{n-1}\right)^{2}-v_{h,-}^{n-1} v_{h,+}^{n-1}\right) d x+\frac{1}{2} \int_{\Sigma_{n}}\left(v_{h,-}^{n}\right)^{2} d x
\end{aligned}
$$

where we have used (2.10) at the last step in the estimates above. Summing over all $Q_{n}$, we obtain

$$
\begin{aligned}
a_{h}\left(v_{h}, v_{h}\right)=\sum_{n=1}^{N} a_{Q_{n}}\left(v_{h}, v_{h}\right) \geq & \sum_{n=1}^{N} \theta_{n} h_{n}\left\|\partial_{t} v_{h}\right\|_{L_{2}\left(Q_{n}\right)}^{2}+\left(1-\frac{\theta_{n} C_{i n v, 0}^{2}}{2}\right)\left\|\nabla_{x} v_{h}\right\|_{L_{2}\left(Q_{n}\right)}^{2} \\
& +\sum_{n=1}^{N} \frac{1}{2}\left\|\llbracket v_{h} \rrbracket^{n-1}\right\|_{L_{2}\left(\Sigma_{n-1}\right)}^{2}+\frac{1}{2}\left\|v_{h,-}^{N}\right\|_{L_{2}\left(\Sigma_{N}\right)}^{2} .
\end{aligned}
$$

Choosing $0<\theta_{n} \leq C_{i n v, 0}^{-2}$, we arrive at (2.11) with $C_{e}=0.5$.

REMARK 2.8. The $V_{0 h}$-ellipticity (2.11) of the bilinear form $a_{h}(\cdot, \cdot)$ implies uniqueness of the solution $u_{h}$ of the IgA scheme (2.9) and, in the finite-dimensional case, uniqueness yields existence. Thus, there always exists a unique IgA solution of the space-time IgA scheme (2.9). Moreover, we can easily derive an a priori estimate. Indeed, using the discrete solution $u_{h}$ as test function in (2.9), the inequalities defined in (2.1) and (2.2) yield

$$
\begin{aligned}
C_{e}\left\|u_{h}\right\|_{d G}^{2} & \leq a_{h}\left(u_{h}, u_{h}\right)=\left|l_{h}\left(u_{h}\right)\right| \\
& \leq\left|\sum_{n=1}^{N} \int_{Q_{n}} f\left(u_{h}^{n}+\theta_{n} h_{n} \partial_{t} u_{h}^{n}\right) d x d t\right|+\left|\int_{\Sigma_{0}} u_{0} u_{h,+}^{0} d x\right| \\
& \leq \sqrt{2} C_{\text {stab }}\left(\|f\|_{L_{2}(Q)}+\left\|u_{0}\right\|_{L_{2}\left(\Sigma_{0}\right)}\right)\left\|u_{h}\right\|_{d G},
\end{aligned}
$$


where $C_{\text {stab }}$ depends on the constant in (2.2) and on $\theta_{\max }=\max _{n}\left\{\theta_{n}\right\}$. Form these estimates, we immediately get the a priori bound

$$
\left\|u_{h}\right\|_{d G} \leq \sqrt{2} C_{s t a b} C_{e}^{-1}\left(\|f\|_{L_{2}(Q)}+\left\|u_{0}\right\|_{L_{2}\left(\Sigma_{0}\right)}\right) .
$$

Later, in the discretization error analysis, we need continuity properties for $a_{h}(\cdot, \cdot)$. Let $V$ and $V_{0 h}$ be the spaces defined in Assumption 2.1 and in (2.6). We define the space $V_{0 h, *}=V+V_{0 h}$ endowed with the norm

$$
\|v\|_{d G, *}=\left(\|v\|_{d G}^{2}+\sum_{n=1}^{N}\left(\theta_{n} h_{n}\right)^{-1}\|v\|_{L_{2}\left(Q_{n}\right)}^{2}+\sum_{n=2}^{N}\left\|v_{-}^{n-1}\right\|_{L_{2}\left(\Sigma_{n-1}\right)}^{2}\right)^{\frac{1}{2}} .
$$

Lemma 2.9. Let $u \in V_{0 h, *}$. Then the boundedness inequality

$$
\left|a_{h}\left(u, v_{h}\right)\right| \leq C_{b}\|u\|_{d G, *}\left\|v_{h}\right\|_{d G}
$$

holds for all $v_{h} \in V_{0 h}$, where $C_{b}=\max \left(C_{i n v, 1} \theta_{\max }, 2\right)$ with $\theta_{\max }=\max _{n}\left\{\theta_{n}\right\} \leq C_{i n v, 0}^{-2}$.

Proof. For the first and the interface jump terms of $a_{h}$, we use (2.12) and (2.1) and obtain

$$
\begin{aligned}
\sum_{n=1}^{N}\left(\int_{Q_{n}} \partial_{t} u v_{h} d x d t+\int_{\Sigma_{n-1}} \llbracket u \rrbracket^{n-1} v_{h,+}^{n-1} d x\right) \\
=-\sum_{n=1}^{N} \int_{Q_{n}} u \partial_{t} v_{h} d x d t+\sum_{n=1}^{N}\left(\int_{\Sigma_{n}} u v_{h,-}^{n} d x-\int_{\Sigma_{n-1}} u v_{h,+}^{n-1} d x\right. \\
\left.+\int_{\Sigma_{n-1}} \llbracket u \rrbracket^{n-1} v_{h,+}^{n-1} d x\right) \\
\leq\left(\sum_{n=1}^{N}\left(\theta_{n} h_{n}\right)^{-1}\left(\int_{Q_{n}} u^{2} d x d t\right)^{2}\right)^{\frac{1}{2}}\left(\sum_{n=1}^{N} \theta_{n} h_{n}\left(\int_{Q_{n}} \partial_{t} v_{h}^{2} d x d t\right)^{2}\right)^{\frac{1}{2}} \\
\quad+\sum_{n=2}^{N} \int_{\Sigma_{n-1}}\left(v_{h,-}^{n-1}-v_{h,+}^{n-1}\right) u_{-}^{n-1} d x+\int_{\Sigma_{N}} v_{h,-}^{n} u_{-}^{n} d x \\
\leq\left(\sum_{n=1}^{N}\left(\theta_{n} h_{n}\right)^{-1}\left(\int_{Q_{n}} u^{2} d x d t\right)^{2}\right)^{\frac{1}{2}}\left(\sum_{n=1}^{N} \theta_{n} h_{n}\left(\int_{Q_{n}} \partial_{t} v_{h}^{2} d x d t\right)^{2}\right)^{\frac{1}{2}} \\
\quad+\left(\sum_{n=2}^{N}\left\|v_{h,-}^{n-1}-v_{h,+}^{n-1}\right\|_{L_{2}\left(\Sigma_{n-1}\right)}^{2}\right)^{\frac{1}{2}}\left(\sum_{n=2}^{N}\left\|u_{-}^{n-1}\right\|_{L_{2}\left(\Sigma_{n-1}\right)}^{2}\right)^{\frac{1}{2}} \\
\quad+\left\|u_{-}^{N}\right\|_{L_{2}\left(\Sigma_{N}\right)}\left\|v_{h,-}^{N}\right\|_{L_{2}\left(\Sigma_{N}\right)} \\
\leq\left(\sum_{n=1}^{N}\left(\theta_{n} h_{n}\right)^{-1}\left(\int_{Q_{n}} u^{2} d x d t\right)^{2}\right)^{\frac{1}{2}}\left(\sum_{n=1}^{N} \theta_{n} h_{n}\left(\int_{Q_{n}} \partial_{t} v_{h}^{2} d x d t\right)^{2}\right)^{\frac{1}{2}} \\
\quad+\sqrt{2}\left(\frac{1}{2} \sum_{n=1}^{N}\left\|\llbracket v_{h} \rrbracket^{n-1}\right\|_{L_{2}\left(\Sigma_{n-1}\right)}^{2}+\frac{1}{2}\left\|v_{h}^{N}\right\|_{L_{2}\left(\Sigma_{N}\right)}^{2}\right)^{\frac{1}{2}} \\
\times \sqrt{2}\left(\sum_{n=2}^{N}\left\|u_{-}^{n-1}\right\|_{L_{2}\left(\Sigma_{n-1}\right)}^{2}+\frac{1}{2}\left\|u_{-}^{N}\right\|_{L_{2}\left(\Sigma_{N}\right)}^{2}\right)^{\frac{1}{2}} .
\end{aligned}
$$


For the second term, an application of Cauchy's inequality yields

$$
\begin{gathered}
\sum_{n=1}^{N} \int_{Q_{n}}\left(\theta_{n} h_{n}\right)^{\frac{1}{2}} \partial_{t} u\left(\theta_{n} h_{n}\right)^{\frac{1}{2}} \partial_{t} v_{h} d x d t+\sum_{n=1}^{N} \int_{Q_{n}} \nabla_{x} u \cdot \nabla_{x} v_{h} d x d t \\
\leq\left(\sum_{n=1}^{N} \theta_{n} h_{n}\left\|\partial_{t} u\right\|_{L_{2}\left(Q_{n}\right)}^{2}\right)^{\frac{1}{2}}\left(\sum_{n=1}^{N} \theta_{n} h_{n}\left\|\partial_{t} v_{h}\right\|_{L_{2}\left(Q_{n}\right)}^{2}\right)^{\frac{1}{2}} \\
+\left(\sum_{n=1}^{N}\left\|\nabla_{x} u\right\|_{L_{2}\left(Q_{n}\right)}^{2}\right)^{\frac{1}{2}}\left(\sum_{n=1}^{N}\left\|\nabla_{x} v_{h}\right\|_{L_{2}\left(Q_{n}\right)}^{2}\right)^{\frac{1}{2}}
\end{gathered}
$$

For the last term, we apply Cauchy's and an inverse inequality to show

$$
\begin{aligned}
& \sum_{n=1}^{N} \int_{Q_{n}} \nabla_{x} u \cdot\left(\theta_{n} h_{n}\right) \nabla_{x} \partial_{t} v_{h} d x d t \\
& \quad \leq\left(\sum_{n=1}^{N}\left\|\nabla_{x} u\right\|_{L_{2}\left(Q_{n}\right)}^{2}\right)^{\frac{1}{2}}\left(\sum_{n=1}^{N}\left(\theta_{n} h_{n}\right)^{2} \sum_{i=1}^{d} \int_{Q_{n}}\left(\partial_{t} \partial_{x_{i}} v_{h}\right)^{2} d x d t\right)^{\frac{1}{2}} \\
& \quad \leq\left(\sum_{n=1}^{N}\left\|\nabla_{x} u\right\|_{L_{2}\left(Q_{n}\right)}^{2}\right)^{\frac{1}{2}}\left(\sum_{n=1}^{N}\left(\theta_{n} h_{n}\right)^{2} C_{i n v, 1} h_{n}^{-2} \sum_{i=1}^{d} \int_{Q_{n}}\left(\partial_{x_{i}} v_{h}\right)^{2} d x d t\right)^{\frac{1}{2}} \\
& \quad \leq C_{\text {inv }, 1} \theta_{\max }\left(\sum_{n=1}^{N}\left\|\nabla_{x} u\right\|_{L_{2}\left(Q_{n}\right)}^{2}\right)^{\frac{1}{2}}\left(\sum_{n=1}^{N}\left\|\nabla_{x} v_{h}\right\|_{L_{2}\left(Q_{n}\right)}^{2}\right)^{\frac{1}{2}}
\end{aligned}
$$

where $\theta_{\max }=\max _{n}\left\{\theta_{n}\right\} \leq C_{i n v, 0}^{-2}$. Gathering together the bounds (2.14), (2.15), and (2.16) and setting $C_{b}=\max \left(C_{i n v, 1} \theta_{\max }, 2\right)$ yield the desired result.

LEMmA 2.10. Let Assumption 2.1 hold, and let $u_{h} \in V_{0 h}$ be the $d G$ IgA solution of (2.9). Then the Galerkin orthogonality

$$
a_{h}\left(u-u_{h}, v_{h}\right)=0
$$

holds for all $v_{h} \in V_{0 h}$.

Proof. Subtracting (2.9) from (2.8) directly yields the Galerkin orthogonality (2.17).

3. A priori discretization error analysis. Based on the quasi-interpolation error estimates presented in $[7,10]$ (see also $[36,45]$ ), we below construct quasi-interpolation operators $\Pi_{h}^{n}: H^{\ell, m}\left(Q_{n}\right) \rightarrow \mathbb{B}_{\Xi_{n}^{d+1}}\left(Q_{n}\right)$, for $n=1, \ldots, N$, suitable for providing anisotropic interpolation error estimates. Utilizing these estimates, we show the desirable anisotropic discretization error estimates at the end of this section.

3.1. Multivariate quasi-interpolants in the parameter domain $\widehat{Q}$. Let $\mathcal{Z}$ be a partition of the interval $I=(0,1)$, i.e., $\mathcal{Z}=\left\{0=z_{1}, z_{2}, \ldots, z_{M}=1\right\}$, with the subintervals $I_{j}=\left(z_{j}, z_{j+1}\right), j=1, \ldots, M-1$, and let $h=\max _{j=1, \ldots, M-1}\left\{z_{j+1}-z_{j}\right\}$ be the maximal mesh size. Based on $\mathcal{Z}$, we consider a knot-vector $\Xi=\left\{0=\xi_{1} \leq \xi_{2} \leq \ldots \leq \xi_{n+p+1}=1\right\}$ and the associated vector of knot multiplicities $\mathcal{M}=\left\{m_{1}, \ldots, m_{M}\right\}$. Let $s=0$ or $s=1$, the integer $\ell$ be such that $0 \leq s \leq \ell \leq p+1$, and let $f \in H^{\ell}(I)$. Due to the quasi-interpolation estimates presented in $[10,45]$, we can construct a quasi-interpolant $\widehat{\Pi}_{h}: H^{\ell}(I) \rightarrow \widehat{\mathbb{B}}_{\Xi}(I)$ such that the interpolation estimate

$$
\left|f-\widehat{\Pi}_{h} f\right|_{H^{s}(I)} \leq C h^{\ell-s}\|f\|_{H^{\ell}(I)}
$$




\section{ETNA}

Kent State University and

Johann Radon Institute (RICAM)

holds, where the positive constant $C$ depends on $p$ and on the uniformity parameters of the partition but not on $h$. The previous construction of the univariate quasi-interpolation can be extended to the multi-dimensional case by means of a tensor-product construction like those presented in Section 2.3. For example, let $f \in H^{\ell}(\widehat{Q})$ with $\ell \geq 1$, and let $\widehat{\Pi}_{\Xi^{k}}$ be the corresponding $k$-th univariate quasi-interpolation operator onto $\widehat{\mathbb{B}}_{\Xi k}$. Then we construct the multi-dimensional B-spline interpolation operator $\widehat{\Pi}_{\Xi^{d+1}}$ by the tensor product

$$
\widehat{\Pi}_{\Xi^{d+1}} f=\bigotimes_{k=1}^{d+1} \widehat{\Pi}_{\Xi^{k}} f .
$$

The general quasi-interpolation properties of the multivariate B-spline interpolants are inherited by the corresponding properties of the univariate interpolants. We refer to $[7,10,45]$ for a comprehensive analysis of the properties of the tensor-product B-spline interpolants.

3.2. Anisotropic quasi-interpolation in space-time patches $\boldsymbol{Q}_{\boldsymbol{n}}$. Let $f \in H^{\ell, m}(Q)$, with $\ell \geq 2$ and $m \geq 1$. As usual, we denote its restriction to the space-time patches $Q_{n}$ by $f^{n}=\left.f\right|_{Q_{n}}$, for $n=1, \ldots, N$. Further, we denote its pull-back function by $\hat{f}^{n}=f \circ \boldsymbol{\Phi}_{n}$. We note that, in general, $\hat{f}^{n}$ does not inherit the regularity of $f$ but rather belongs to a bent Sobolev space $\mathcal{H}^{\ell}(\widehat{Q})=\mathcal{H}^{\ell_{1}}(I) \otimes \ldots \otimes \mathcal{H}^{\ell_{d}}(I) \otimes \mathcal{H}^{\ell_{d+1}}(I)$, which allows less regularity across the microelement interfaces, where $\mathcal{H}^{\ell_{1}}(I), \ldots, \mathcal{H}^{\ell_{d+1}}(I)$ are the corresponding univariate bent-Sobolev spaces; see [7, 10]. To derive anisotropic quasi-interpolation estimates, we have strongly been inspired by the results presented in [10,11], which are also suitable for anisotropic meshes. The generalization here is that we provide anisotropic interpolation estimates that follow the anisotropic regularity of the solution. In the spirit of (3.1), we define the interpolant $\widehat{\Pi}_{\Xi_{n}^{d+1}} \hat{f}^{n}$ of the pull-back $\hat{f}^{n}$ in the parameter domain $\widehat{Q}$. For simplicity, we shall write $\widehat{\Pi}_{h}^{n}$ instead of $\widehat{\Pi}_{\Xi_{n}^{d+1}}$. Accordingly, we define the quasi-interpolant of $f^{n}$ as $\Pi_{h}^{n} f^{n}=\left(\widehat{\Pi}_{h}^{n} \hat{f}^{n}\right) \circ \boldsymbol{\Phi}_{n}^{-1}$ in the physical space-time patches $Q_{n}$. By extension, we can define the global interpolant $\Pi_{h}: H^{\ell, m}(Q) \rightarrow V_{h}$ as $\left.\left(\Pi_{h} f\right)\right|_{Q_{n}}=\Pi_{h}^{n} f^{n}$; see, e.g., [10].

Before stating estimates on how well $\Pi_{h} f$ approximates $f \in H^{\ell, m}(Q)$, some terminology is required. We recall the definition of the differential operator

$$
\partial^{(\boldsymbol{\alpha}, m)} f:=\partial^{\left(\alpha_{1}, \ldots, \alpha_{d}, m\right)} f=\frac{\partial^{\alpha_{1}} \ldots \partial^{\alpha_{d}} \partial^{m}}{\partial x_{1}^{\alpha_{1}} \ldots \partial x_{d}^{\alpha_{d}} \partial t^{m}} f
$$

from Section 2.1. In order to derive the anisotropic estimates, we have to introduce the derivatives with respect to the coordinate system that is naturally induced by the mappings $\boldsymbol{\Phi}_{n}: \widehat{Q} \rightarrow Q_{n}$; see (2.5). We again note that the mappings $\boldsymbol{\Phi}_{n}$ are constructed on relatively coarse meshes and are highly smooth (polynomials) on the microelements of those meshes.

We recall that the columns of the Jacobian matrix of $\boldsymbol{\Phi}_{n}$ (see (2.5) and Assumption 2.4) have the form

$$
\left[\frac{\partial \Phi_{n, 1}}{\partial \hat{x}_{i}}, \ldots, \frac{\partial \Phi_{n, d}}{\partial \hat{x}_{i}}, \frac{\partial \Phi_{n, d+1}}{\partial \hat{x}_{i}}\right]^{\top}=\left[\frac{\partial \Phi_{n, 1}}{\partial \hat{x}_{i}}, \ldots, \frac{\partial \Phi_{n, d}}{\partial \hat{x}_{i}}, 0\right]^{\top},
$$

where we have used the fact that $\frac{\partial \Phi_{n, d+1}}{\partial \hat{x}_{i}}=0$, for $i=1, \ldots, d$, which easily follows from the tensor-product construction of each $\boldsymbol{\Phi}_{n}$. We now introduce the notation

$$
\mathbf{g}_{n, i}(x, t)=\left[\frac{\partial \Phi_{n, 1}}{\partial \hat{x}_{i}}\left(\boldsymbol{\Phi}_{n}^{-1}(x, t)\right), \ldots, \frac{\partial \Phi_{n, d}}{\partial \hat{x}_{i}}\left(\boldsymbol{\Phi}_{n}^{-1}(x, t)\right), 0\right]^{\top} .
$$


Then the derivatives of $f$ with respect to the spatial $\boldsymbol{\Phi}_{n}$-coordinates are given as follows: the first derivatives are just the directional derivatives with respect to $\mathbf{g}_{n, i}$ for $i=1, \ldots, d$, i.e.,

$$
\begin{gathered}
\frac{\partial f(x, t)}{\partial \mathbf{g}_{n, 1}}=\nabla f(x, t) \cdot \mathbf{g}_{n, 1}(x, t), \\
\vdots \\
\frac{\partial f(x, t)}{\partial \mathbf{g}_{n, d}}=\nabla f(x, t) \cdot \mathbf{g}_{n, d}(x, t),
\end{gathered}
$$

whereas the "one-directional" high-order derivatives are given by

$$
\frac{\partial^{\alpha_{i}} f}{\partial \mathbf{g}_{n, i}^{\alpha_{i}}}=\underbrace{\frac{\partial}{\partial \mathbf{g}_{n, i}}\left(\ldots\left(\frac{\partial f}{\partial \mathbf{g}_{n, i}}\right)\right)}_{\alpha_{i} \text {-times }} .
$$

Let the multi-index $\boldsymbol{\alpha}=\left(\alpha_{1}, \ldots, \alpha_{d}\right)$ be defined as in Section 2.1. To deal with multi-direction derivatives, we introduce the notations $\mathbf{x}^{\boldsymbol{\alpha}, q}=x_{1}^{\alpha_{1}} \ldots, x_{d}^{\alpha_{d}}, x_{d+1}^{q}$, where $\mathbf{x} \in \mathbb{R}^{d+1}, q \in \mathbb{N}_{0}$,

$$
D_{\mathbf{\Phi}_{n}}^{\boldsymbol{\alpha}} f=\frac{\partial^{\alpha_{1}}}{\partial \mathbf{g}_{n, 1}^{\alpha_{1}}} \ldots \frac{\partial^{\alpha_{d}} f}{\partial \mathbf{g}_{n, d}^{\alpha_{d}}}, \quad \text { and } \quad D_{\boldsymbol{\Phi}_{n}}^{\boldsymbol{\alpha}, q} f=\frac{\partial^{\alpha_{1}}}{\partial \mathbf{g}_{n, 1}^{\alpha_{1}}} \ldots \frac{\partial^{\alpha_{d}}}{\partial \mathbf{g}_{n, d}^{\alpha_{d}}} \frac{\partial^{q} f}{\partial \mathbf{g}_{n, d+1}^{q}} .
$$

In relation to the derivatives $D_{\boldsymbol{\Phi}_{n}}^{\boldsymbol{\alpha}, q} f$, we define the norms and seminorms

and

$$
\|f\|_{\mathcal{H}_{\boldsymbol{\Phi}_{n}}^{\boldsymbol{\alpha}, q}\left(Q_{n}\right)}^{2}=\sum_{s_{1}=0}^{\alpha_{1}} \ldots \sum_{s_{d}=0}^{\alpha_{d}} \sum_{s_{d+1}=0}^{q}|f|_{\mathcal{H}_{\boldsymbol{\Phi}_{n}}^{\boldsymbol{\alpha}, q}\left(Q_{n}\right)}^{2}
$$

$$
|f|_{\mathcal{H}_{\Phi_{n}}^{\alpha, q}\left(Q_{n}\right)}^{2}=\sum_{E \in T_{h_{n}}^{n}\left(Q_{n}\right)}|f|_{H_{\Phi_{n}}^{\alpha, q}(E)}^{2},
$$

respectively, where $|f|_{H_{\mathbf{\Phi}_{n}(E)}^{\boldsymbol{\alpha}, q}\left({ }^{2}\right.}^{2}=\left\|D_{\mathbf{\Phi}_{n}}^{\boldsymbol{\alpha}, q} f\right\|_{L_{2}(E)}$. We now introduce the space $H_{\mathbf{\Phi}_{n}}^{\boldsymbol{\alpha}, q}\left(Q_{n}\right)$ endowed with the norm $\|\cdot\|_{H_{\Phi, n}^{\alpha, q}\left(Q_{n}\right)}=\|\cdot\|_{\mathcal{H}_{\Phi_{n}}^{\alpha, q}\left(Q_{n}\right)}$. Below, we show a relation between the norms based on the $\mathbf{g}_{n, i}$-directional derivatives and those based on the usual partial derivatives.

Proposition 3.1. Let $f: Q \rightarrow \mathbb{R}$ be a smooth function, and let Assumption 2.4 hold. Then, for $|\boldsymbol{\alpha}|=1$ and for all $E \in T_{h_{n}}^{n}\left(Q_{n}\right)$, we have the equivalence relations

$$
\left\|\partial^{\boldsymbol{\alpha}} f\right\|_{L_{2}(E)} \sim \sum_{|\boldsymbol{\alpha}|=1}\left\|D_{\mathbf{\Phi}_{n}}^{\boldsymbol{\alpha}, 0} f\right\|_{L_{2}(E)} \quad \text { and } \quad\|f\|_{H^{\ell, m}(E)} \sim \sum_{|\boldsymbol{\alpha}|=1}\|f\|_{\mathcal{H}_{\boldsymbol{\Phi}_{n}}^{\ell \boldsymbol{\alpha}, m}(E)},
$$

where the associated constants depend on $p, \gamma, \mathbf{g}_{n, i}$, and $\mathbf{\Phi}_{n}$.

Proof. The inequalities (3.5) immediately follow from (3.2a), (3.3), (3.4), and (3.2).

Assumption 3.2. For simplicity, we assume that $p+1 \geq \max (\ell, m)$; cf. also Assumption 2.1.

TheOrem 3.3. Let the Assumptions 2.2, 2.4, and 3.2 hold. Let $E \in T_{h_{n}}^{n}\left(Q_{n}\right)$ and $\widetilde{E}$ be its support extension. Furthermore, let $f \in H^{\ell, m}\left(Q_{n}\right)$ with $\ell \geq 2$ and $m \geq 1$. Then the quasi-interpolation estimates

$$
\begin{gathered}
\left(\sum_{E \in T_{h_{n}}^{n}\left(Q_{n}\right)}\left|\nabla_{x}\left(f-\Pi_{h}^{n} f\right)\right|_{L_{2}(E)}^{2}\right)^{\frac{1}{2}} \leq C_{x}\left(h_{n}^{\ell-1}+h_{n}^{m}\right)\|f\|_{H^{\ell, m}\left(Q_{n}\right)}, \\
\left(\sum_{E \in T_{h_{n}}^{n}\left(Q_{n}\right)}\left|\partial_{t}\left(f-\Pi_{h}^{n} f\right)\right|_{L_{2}(E)}^{2}\right)^{\frac{1}{2}} \leq C_{t}\left(h_{n}^{\ell}+h_{n}^{m-1}\right)\|f\|_{H^{\ell, m}\left(Q_{n}\right)}, \\
\left(\sum_{E \in T_{h_{n}}^{n}\left(Q_{n}\right)}\left|f-\Pi_{h}^{n} f\right|_{L_{2}(E)}^{2}\right)^{\frac{1}{2}} \leq C_{0}\left(h_{n}^{\ell}+h_{n}^{m}\right)\|f\|_{H^{\ell, m}\left(Q_{n}\right)}
\end{gathered}
$$


hold, where the positive constants $C_{x}, C_{t}$, and $C_{0}$ only depend on $d, p, \gamma, \mathbf{g}_{n, i}$, and $\mathbf{\Phi}_{n}$.

Proof. We already noted above that the last component of $\mathbf{g}_{n, i}$ is equal to zero. This implies that the derivatives $\frac{\partial f(x, t)}{\partial \mathbf{g}_{n, i}}$ do not include terms like $\frac{\partial f}{\partial t}$; see (3.2). Since $f \in H^{\ell, m}(Q)$, we conclude that $f \in H_{\mathbf{\Phi}_{n}, 0}^{\ell \boldsymbol{\alpha}, 0}\left(Q_{n}\right) \cap H_{\mathbf{\Phi}_{n}}^{0, m}\left(Q_{n}\right)$, where $\boldsymbol{\alpha}$ is a multi-index with $d$-components such that $|\boldsymbol{\alpha}|=1$. Making use of the interpolation results presented in [10] (see Theorem 4.18), we arrive at the estimate

$$
\left\|D_{\boldsymbol{\Phi}_{n}}^{\boldsymbol{\alpha}, 0}\left(f-\Pi_{h}^{n} f\right)\right\|_{L_{2}(E)} \leq c_{1}\left(h_{n}^{\ell-1}+h_{n}^{m}\right) \sum_{|\boldsymbol{\alpha}|=1}\|f\|_{\mathcal{H}_{\boldsymbol{\Phi}_{n}}^{\ell \boldsymbol{\alpha}, m}(\widetilde{E})} .
$$

Using (3.5) and (3.7), we can derive the interpolation error estimate

$$
\begin{aligned}
\left\|\nabla_{x}\left(f-\Pi_{h}^{n} f\right)\right\|_{L_{2}(E)} & \leq c_{2} \sum_{|\boldsymbol{\alpha}|=1}\left\|D_{\mathbf{\Phi}_{n}}^{\boldsymbol{\alpha}, 0}\left(f-\Pi_{h}^{n} f\right)\right\|_{L_{2}(E)} \\
& \leq c_{3}\left(h_{n}^{\ell-1}+h_{n}^{m}\right)\|f\|_{H^{\ell, m}(\widetilde{E})},
\end{aligned}
$$

with positive constants $c_{2}$ and $c_{3}$ depending on $d, p, \gamma$, and $\boldsymbol{\Phi}_{n}$. Summing over all $E$ in $T_{h_{n}}^{n}\left(Q_{n}\right)$, the estimate (3.8) yields

$$
\begin{aligned}
\sum_{E}\left\|\nabla_{x}\left(f-\Pi_{h}^{n} f\right)\right\|_{L_{2}(E)}^{2} & \leq c_{4}\left(h_{n}^{\ell-1}+h_{n}^{m}\right)^{2} \sum_{E}\|f\|_{H^{\ell, m}(\widetilde{E})}^{2} \\
& \leq c_{5}\left(h_{n}^{\ell-1}+h_{n}^{m}\right)^{2} \sum_{E} \sum_{E^{\prime} \in \widetilde{E}}\|f\|_{H^{\ell, m}\left(E^{\prime}\right)}^{2}
\end{aligned}
$$

where the constants $c_{4}$ and $c_{5}$ only depend on the constant $c_{3}$. Now, we observe that the last double sum in (3.9) consists of repeated element norm terms of the form $\|f\|_{H^{\ell, m}(E)}$. More precisely, for every element $E \in T_{h_{n}}^{n}\left(Q_{n}\right)$, the related norm term $\|f\|_{H^{\ell, m}(E)}$ appears as many times in (3.9) as the number of the extension supports $\widetilde{E}$, lets say $E_{N b, \widetilde{E}}$, to which the element $E$ belongs. Due to the construction procedure of B-splines, $E_{N b, \widetilde{E}}$ depends on the underlying B-spline degree and the knot repetitions $m_{i}$, i.e., the smoothness of the B-splines across the microelement interfaces. Setting $E_{\max , \widetilde{E}}=\max _{E \in T_{h_{n}}^{n}\left(Q_{n}\right)}\left\{E_{N b, \widetilde{E}}\right\}$, the inequality (3.9) gives

$$
\begin{aligned}
\sum_{E}\left\|\nabla_{x}\left(f-\Pi_{h}^{n} f\right)\right\|_{L_{2}(E)}^{2} & \leq c_{5} E_{\max , \widetilde{E}}\left(h_{n}^{\ell-1}+h_{n}^{m}\right)^{2} \sum_{E}\|f\|_{H^{\ell, m}(E)}^{2} \\
& \leq C_{x}^{2}\left(h_{n}^{\ell-1}+h_{n}^{m}\right)^{2}\|f\|_{H^{\ell, m}\left(Q_{n}\right)}^{2},
\end{aligned}
$$

and the estimate (3.6a) follows. Following a similar procedure, we can show the estimates (3.6b) and (3.6c).

THEOREM 3.4. Let $\ell \geq 2$ and $m \geq 1$ be integers, and let $f \in H^{\ell, m}(Q)$. Furthermore, let the Assumptions 2.2, 2.4, and 3.2 hold, and let $\Pi_{h}^{n} f$ be the corresponding quasi-interpolant defined above. Then there exist constants $C_{1}^{*}$ and $C_{2}^{*}$ independent of $f$ and $h$ but dependent on the constants from (2.10) and (3.6) such that the following quasi-interpolation error estimates

$$
\begin{aligned}
\left\|f-\Pi_{h}^{n} f\right\|_{L_{2}\left(\partial Q_{n}\right)} & \leq C_{1}^{*}\left(h_{n}^{\ell-\frac{1}{2}}+h_{n}^{m-\frac{1}{2}}\right)\|f\|_{H^{\ell, m}\left(Q_{n}\right)}, \\
\left\|f-\Pi_{h} f\right\|_{d G, *} & \leq C_{2}^{*}\left(h^{\ell-1}+h^{m-\frac{1}{2}}\right)\|f\|_{H^{\ell, m}(Q)}
\end{aligned}
$$

hold. 
Proof. Using first the trace inequalities given in (2.10) and then (3.6), we get

$$
\begin{aligned}
\left\|f-\Pi_{h}^{n} f\right\|_{L_{2}\left(\partial Q_{n}\right)}^{2} & \leq C_{t r}\left(h_{n}^{-1}\left(\left\|f-\Pi_{h}^{n} f\right\|_{L_{2}\left(Q_{n}\right)}^{2}+h_{n}\left\|\nabla f-\nabla \Pi_{h}^{n} f\right\|_{L_{2}\left(Q_{n}\right)}^{2}\right)\right) \\
& \leq\left(C_{1}^{*}\right)^{2}\left(h_{n}^{2\left(\ell-\frac{1}{2}\right)}+h_{n}^{2\left(m-\frac{1}{2}\right)}\right)\|f\|_{H^{\ell, m}\left(Q_{n}\right)}^{2} .
\end{aligned}
$$

Recalling the definition of $\|\cdot\|_{d G, *}$ and using again (3.6) as well as the just proven estimate (3.10a), we obtain

$$
\begin{aligned}
\left\|f-\Pi_{h} f\right\|_{d G, *}^{2}= & \sum_{n=1}^{N}\left(\left\|\nabla_{x}\left(f-\Pi_{h}^{n} f\right)\right\|_{L_{2}\left(Q_{n}\right)}^{2}+\theta_{n} h_{n}\left\|\partial_{t}\left(f-\Pi_{h}^{n} f\right)\right\|_{L_{2}\left(Q_{n}\right)}^{2}\right. \\
& \left.+\frac{1}{2}\left\|\llbracket\left(f-\Pi_{h} f\right)^{n-1} \rrbracket\right\|_{L_{2}\left(\Sigma_{n-1}\right)}^{2}\right) \\
& +\frac{1}{2}\left\|f-\Pi_{h}^{N} f\right\|_{L_{2}\left(\Sigma_{N}\right)}^{2}+\sum_{n=1}^{N} \frac{1}{\theta_{n} h_{n}}\left\|f-\Pi_{h}^{n} f\right\|_{L_{2}\left(Q_{n}\right)}^{2} \\
& +\sum_{n=2}^{N}\left\|\left(f-\Pi_{h}^{n-1} f\right)_{-}^{n-1}\right\|_{L_{2}\left(\Sigma_{n-1}\right)}^{2} \\
\leq & \sum_{n=1}^{N}\left(C_{0, n}\left(h_{n}^{2(\ell-1)}+h_{n}^{2\left(m-\frac{1}{2}\right)}\right)+C_{1, n}\left(h_{n}^{2\left(\ell-\frac{1}{2}\right)}+h_{n}^{2\left(m-\frac{1}{2}\right)}\right)\right. \\
\leq & \left.+\sum_{n=1}^{N} C_{n}\left(h_{n}^{2(\ell-1)}+\theta_{n} h_{n}^{2 \ell-1}+h_{n}^{2 m-1}+\theta_{n} h_{n}^{2 m-1}\right)\|f\|_{H^{\ell, m}\left(Q_{n}\right)}^{2}\left(h_{n}^{2 \ell}+h_{n}^{2 m}\right)\right)\|f\|_{H^{\ell, m}\left(Q_{n}\right)}^{2} \\
\leq & \left(C_{2}^{*}\right)^{2}\left(h^{2(\ell-1)}+h^{2\left(m-\frac{1}{2}\right)}\right)\|f\|_{H^{\ell, m}(Q)}^{2},
\end{aligned}
$$

where $C_{n}=C_{0, n}+C_{1, n}+C_{2, n}$ and $\left(C_{2}^{*}\right)^{2}=\max _{n=1, \ldots, N}\left\{\left(1+\theta_{n}\right) C_{n}\right\}$. We mention that a reduction of the terms $h^{\ell-1}$ and $h^{\ell-\frac{1}{2}}$ has been performed. This completes the proof of (3.10b).

3.3. Discretization error estimate. We are now in the position to prove the discretization error estimate in the dG-norm $\|\cdot\|_{d G}$.

THEOREM 3.5. Let $u$ and $u_{h}$ solve (2.3) and (2.9), respectively. Under Assumption 2.1, there exist a positive generic constant $C$, which is independent of $h$, such that

$$
\left\|u-u_{h}\right\|_{d G} \leq C\left(h^{\ell-1}+h^{m-\frac{1}{2}}\right)\|u\|_{H^{\ell, m}(Q)} .
$$

Moreover, if $1 \leq m<\ell \leq p+1$, then

$$
\left\|u-u_{h}\right\|_{d G} \leq C h^{m-\frac{1}{2}}\|u\|_{H^{\ell, m}(Q)} .
$$

Proof. Using the $V_{0 h}$ ellipticity (2.11) and the boundedness estimate (2.13) as well as the consistency (2.17), we obtain

$$
\begin{aligned}
\left\|u_{h}-\Pi_{h} u\right\|_{d G}^{2} & \leq C_{e}^{-1} a_{h}\left(u_{h}-\Pi_{h} u, u_{h}-\Pi_{h} u\right)=C_{e}^{-1} a_{h}\left(u-\Pi_{h} u, u_{h}-\Pi_{h} u\right) \\
& \leq\left(C_{b} / C_{e}\right)\left\|u-\Pi_{h} u\right\|_{d G, *}\left\|u_{h}-\Pi_{h} u\right\|_{d G} .
\end{aligned}
$$


Hence, applying the triangle inequality $\left\|u-u_{h}\right\|_{d G} \leq\left\|u-\Pi_{h} u\right\|_{d G, *}+\left\|u_{h}-\Pi_{h} u\right\|_{d G}$, we derive

$$
\left\|u-u_{h}\right\|_{d G} \leq\left(1+\left(C_{b} / C_{e}\right)\right)\left\|u-\Pi_{h} u\right\|_{d G, *} .
$$

Utilizing estimate (3.10) in (3.13) yields (3.11). Estimate (3.12) is a direct result of (3.11). 口

REMARK 3.6. We remark that for the case of highly smooth solutions, i.e., when $p+1 \leq \min (\ell, m)$, the estimate (3.11) takes the form

$$
\left\|u-u_{h}\right\|_{d G} \leq C h^{p}\|u\|_{H^{p+1, p+1}(Q)} .
$$

4. Matrix representation and space-time multigrid. We recall the discrete variational problem given in (2.9), where we want to find $u_{h} \in V_{0 h}$ such that

$$
a_{h}\left(u_{h}, v_{h}\right)=l_{h}\left(v_{h}\right), \quad \forall v_{h} \in V_{0 h},
$$

with $V_{0 h}:=V_{0 h}^{1} \times \ldots \times V_{0 h}^{N}$ and

$$
a_{h}\left(u_{h}, v_{h}\right)=\sum_{n=1}^{N} a_{Q_{n}}\left(u_{h}, v_{h}\right),
$$

where the local bilinear form for each space-time patch $n=1, \ldots, N$ is given by

$$
\begin{aligned}
a_{Q_{n}}\left(u_{h}, v_{h}\right)= & \int_{Q_{n}} \partial_{t} u_{h}^{n}\left(v_{h}^{n}+\theta_{n} h_{n} \partial_{t} v_{h}^{n}\right)+\nabla_{x} u_{h}^{n} \cdot \nabla_{x}\left(v_{h}^{n}+\theta_{n} h_{n} \partial_{t} v_{h}^{n}\right) d x d t \\
& \quad+\int_{\Sigma_{n-1}} \llbracket u_{h} \rrbracket^{n-1} v_{h,+}^{n-1} d s \\
= & \int_{Q_{n}} \partial_{t} u_{h}^{n}\left(v_{h}^{n}+\theta_{n} h_{n} \partial_{t} v_{h}^{n}\right)+\nabla_{x} u_{h}^{n} \cdot \nabla_{x}\left(v_{h}^{n}+\theta_{n} h_{n} \partial_{t} v_{h}^{n}\right) d x d t \\
& \quad+\int_{\Sigma_{n-1}} u_{h,+}^{n-1} v_{h,+}^{n-1} d s-\int_{\Sigma_{n-1}} u_{h,-}^{n-1} v_{h,+}^{n-1} d s \\
= & : b_{Q_{n}}\left(u_{h}^{n}, v_{h}^{n}\right)-\int_{\Sigma_{n-1}} u_{h,-}^{n-1} v_{h,+}^{n-1} d s .
\end{aligned}
$$

For the local spaces $V_{0 h}^{n}$ defined by (2.7), we now introduce the simpler notation $\varphi_{j}^{n}$ for the $\mathrm{B}$-spline basis functions such that

$$
V_{0 h}^{n}=\operatorname{span}\left\{\varphi_{j}^{n}\right\}_{j=1}^{N_{n}},
$$

for $n=1, \ldots, N$. Then, from the discrete problem (2.9), we obtain the linear system

$$
\mathbf{L}_{h} \underline{u}_{h}:=\left[\begin{array}{ccccc}
\mathbf{A}_{1} & & & & \\
-\mathbf{B}_{2} & \mathbf{A}_{2} & & & \\
& -\mathbf{B}_{3} & \mathbf{A}_{3} & & \\
& & \ddots & \ddots & \\
& & & -\mathbf{B}_{N} & \mathbf{A}_{N}
\end{array}\right]\left[\begin{array}{c}
\underline{u}_{1} \\
\underline{u}_{2} \\
\underline{u}_{3} \\
\vdots \\
\underline{u}_{N}
\end{array}\right]=\left[\begin{array}{c}
\underline{f}_{1} \\
\underline{f}_{2} \\
\underline{f}_{3} \\
\vdots \\
\underline{f}_{N}
\end{array}\right]=: \underline{f}_{h},
$$

with the matrices

$$
\mathbf{A}_{n}[i, j]:=b_{Q_{n}}\left(\varphi_{j}^{n}, \varphi_{i}^{n}\right), \quad \text { for } i, j=1, \ldots, N_{n},
$$


on the diagonal for $n=1, \ldots, N$, and the matrices

$$
\mathbf{B}_{n}[i, k]:=\int_{\Sigma_{n-1}} \varphi_{k,-}^{n-1} \varphi_{i,+}^{n-1} d s, \quad \text { for } k=1, \ldots, N_{n-1} \text { and } i=1, \ldots, N_{n},
$$

on the lower off-diagonal for $n=2, \ldots, N$. Moreover, the right-hand sides are given by

$$
\underline{f}_{n}[i]:=l_{h}\left(\varphi_{i}^{n}\right), \quad i=1, \ldots, N_{n},
$$

for $n=1, \ldots, N$. The linear system (4.1) can be solved by solving the local space-time problems sequentially from one space-time patch to the next space-time patch, i.e., as for a time-stepping scheme

$$
\mathbf{A}_{n} \underline{u}_{n}=\underline{f}_{n}+\mathbf{B}_{n} \underline{u}_{n-1} \quad \text { for } n=2, \ldots, N .
$$

In this work, we will solve the linear system (4.1) by using a space-time multigrid approach similar to the one proposed in [20]. In particular, we apply an (inexact) damped Jacobi scheme as smoother, i.e.,

$$
\underline{u}_{h}^{k+1}=\underline{u}_{h}^{k}+\omega \mathbf{D}_{h}^{-1}\left[\underline{f}_{h}-\mathbf{L}_{h} \underline{u}_{h}^{k}\right] \quad \text { for } k=1,2, \ldots,
$$

where we use the block diagonal matrix $\mathbf{D}_{h}:=\operatorname{diag}\left\{\mathbf{A}_{n}\right\}_{n=1}^{N}$ and the damping parameter $\omega=\frac{1}{2}$; see also [20]. We speed up the application of the smoothing iteration by replacing the exact inverse of $\mathbf{D}_{h}$ by some appropriate approximation. In detail, we will apply one V-cycle iteration of an algebraic multigrid solver (hypre [17, 18]) with respect to the diagonal matrices $\mathbf{A}_{n}, n=1, \ldots, N$, i.e., for each single space-time patch $Q_{n}$. For the single patch case, this type of solvers where successfully used in [34]. For the space-time multigrid approach, we construct a space-time hierarchy by always combining two space-time patches to one coarser space-time patch, where we always apply standard coarsening in the time and space directions. We then have all the components available for setting up a standard multigrid V-cycle. The advantage is that this method is fully parallel with respect to space and time since we use an additive smoother in time and apply standard parallel solvers in space. Moreover, we will use one iteration of this space-time multigrid V-cycle as a preconditioner for the GMRES method.

If the IgA maps $\boldsymbol{\Phi}_{n}: \widehat{Q} \rightarrow Q_{n}, n=1, \ldots, N$, preserve the tensor-product structure of the IgA basis functions $\varphi_{i}^{n}$, then we can use this information to save assembling time and storage costs for the linear system (4.1). In this case, we can write the basis functions $\varphi_{i}^{n}$ in the form

$$
\varphi_{i}^{n}(x, t)=\phi_{i_{x}}^{n}(x) \psi_{i_{t}}^{n}(t), \quad \text { with } i_{x} \in\left\{1, \ldots, N_{n, x}\right\} \text { and } i_{t} \in\left\{1, \ldots, N_{n, t}\right\},
$$

where $N_{n}=N_{n, x} N_{n, t}$. Using this representation, we can write the matrices $\mathbf{A}_{n}$, for $n=1, \ldots, N$, as

$$
\mathbf{A}_{n}=\mathbf{M}_{n, x} \otimes \mathbf{K}_{n, t}+\mathbf{K}_{n, x} \otimes \mathbf{M}_{n, t},
$$

with the standard mass and stiffness matrices with respect to space

$$
\mathbf{M}_{n, x}\left[i_{x}, j_{x}\right]:=\int_{\Omega} \phi_{j_{x}}^{n} \phi_{i_{x}}^{n} d x, \quad \mathbf{K}_{n, x}\left[i_{x}, j_{x}\right]:=\int_{\Omega} \nabla_{x} \phi_{j_{x}}^{n} \cdot \nabla_{x} \phi_{i_{x}}^{n} d x,
$$

where $i_{x}, j_{x}=1, \ldots, N_{n, x}$, and the corresponding matrices with respect to time

$$
\begin{aligned}
\mathbf{K}_{n, t}\left[i_{t}, j_{t}\right] & :=\int_{t_{n-1}}^{t_{n}} \partial_{t} \psi_{j_{t}}^{n}\left(\psi_{i_{t}}^{n}+\theta_{n} h_{n} \partial_{t} \psi_{i_{t}}^{n}\right) d t+\psi_{j_{t}}^{n}\left(t_{n-1}\right) \psi_{i_{t}}^{n}\left(t_{n-1}\right), \\
\mathbf{M}_{n, t}\left[i_{t}, j_{t}\right] & :=\int_{t_{n-1}}^{t_{n}} \psi_{j_{t}}^{n}\left(\psi_{i_{t}}^{n}+\theta_{n} h_{n} \partial_{t} \psi_{i_{t}}^{n}\right) d t,
\end{aligned}
$$




\section{ETNA}

Kent State University and

Johann Radon Institute (RICAM)

TABLE 5.1

Error in the $d G$-norm and convergence rates in terms of eoc for $B$-spline degrees 2,3 , and 4 .

\begin{tabular}{c|cc|cc|cc} 
& \multicolumn{2}{|c|}{$p=2$} & \multicolumn{2}{|c}{$p=3$} & \multicolumn{2}{c}{$p=4$} \\
refinement & error & eoc & error & eoc & error & eoc \\
\hline 0 & $2.78183 \mathrm{E}-01$ & - & $3.75765 \mathrm{E}-02$ & - & $8.82743 \mathrm{E}-03$ & - \\
1 & $5.65687 \mathrm{E}-02$ & 2.30 & $7.06373 \mathrm{E}-03$ & 2.41 & $7.69525 \mathrm{E}-04$ & 3.51 \\
2 & $1.34029 \mathrm{E}-02$ & 2.08 & $8.08757 \mathrm{E}-04$ & 3.12 & $4.58646 \mathrm{E}-05$ & 4.07 \\
3 & $3.30531 \mathrm{E}-03$ & 2.02 & $9.84041 \mathrm{E}-05$ & 3.04 & $2.89757 \mathrm{E}-06$ & 3.98 \\
4 & $8.23479 \mathrm{E}-04$ & 2.01 & $1.22122 \mathrm{E}-05$ & 3.01 & $1.83866 \mathrm{E}-07$ & 3.98 \\
5 & $2.05689 \mathrm{E}-04$ & 2.00 & $1.52369 \mathrm{E}-06$ & 3.00 & $1.16102 \mathrm{E}-08$ & 3.99 \\
6 & $5.14104 \mathrm{E}-05$ & 2.00 & $1.90371 \mathrm{E}-07$ & 3.00 & $7.29856 \mathrm{E}-10$ & 3.99 \\
7 & $1.28518 \mathrm{E}-05$ & 2.00 & $2.37934 \mathrm{E}-08$ & 3.00 & $4.83346 \mathrm{E}-11$ & 3.92
\end{tabular}

with $i_{t}, j_{t}=1, \ldots, N_{n, t}$. The matrices on the off-diagonal $\mathbf{B}_{n}, n=2, \ldots, N$, can be written in the form

$$
\mathbf{B}_{n}:=\widetilde{\mathbf{M}}_{n, x} \otimes \mathbf{N}_{n, t}
$$

with the matrices

$$
\widetilde{\mathbf{M}}_{n, x}\left[i_{x}, k_{x}\right]:=\int_{\Omega} \phi_{k_{x}}^{n-1} \phi_{i_{x}}^{n} d x \quad \text { and } \quad \mathbf{N}_{n, t}\left[i_{t}, k_{t}\right]:=\psi_{k_{t}}^{n-1}\left(t_{n-1}\right) \psi_{i_{t}}^{n}\left(t_{n-1}\right),
$$

where $i_{x}=1, \ldots, N_{n, x}, k_{x}=1, \ldots, N_{n-1, x}, i_{t}=1, \ldots, N_{n, t}$, and $k_{t}=1, \ldots, N_{n-1, t}$.

5. Numerical examples. In the following, we present numerical examples supporting the theory developed in this paper. In Section 5.1, we verify the a priori error estimate from Theorem 3.5 for higher-order B-splines. In Section 5.2, we show the parallel performance of the space-time solver introduced in Section 4.

5.1. Convergence studies. In the first example, the diffusion problem is considered in the two-dimensional space-time cylinder $Q=\Omega \times(0,4)$ with $\Omega=(0,1)$. We choose homogeneous boundary conditions and the source function

$$
f(x, t)=\pi \sin (\pi x)\left(\frac{1}{2} \cos \left(\frac{\pi}{2}(t+1)\right)+\pi \sin \left(\frac{\pi}{2}(t+1)\right)\right)
$$

resulting from the exact solution $u(x, t)=\sin (\pi x) \sin \left(\frac{\pi}{2}(t+1)\right)$. The space-time cylinder $Q$ is decomposed into four space-time patches $Q_{n}=\Omega \times\left(t_{n-1}, t_{n}\right)$ where the partition in time is given by $\left\{t_{0}, t_{1}, t_{2}, t_{3}, t_{4}\right\}=\{0,1,2,3,4\}$; see Fig. 5.1(a). The problem has been solved on a sequence of meshes obtained from an initial mesh by uniform refinements; see also Fig. 5.1(a).

According to Figure 5.1(a), the mesh on $Q_{1}$ and $Q_{3}$ has one additional refinement. We discretize the problem using B-splines of degree $p=\{2,3,4\}$ and choose $\theta_{n}=0.2$ for all space-time patches. The final linear system (4.1) is solved by means of a direct solver, where we used the PARDISO 5.0.0 Solver Project [42, 43]. The algorithm is realized in the isogeometric open source $\mathrm{C}++$ library $\mathrm{G}+\mathrm{SMO}^{1}$. The solution $u_{h}$ on a coarse mesh with $h=0.25$ is visualized in Figure 5.1(a). The error in the dG-norm and the convergence rates in terms of eoc are presented in Table 5.1 and plotted in Figure 5.1(b). We observe that the obtained experimental order of convergence (eoc), defined by eoc $=\ln \left(e_{i-1} / e_{i}\right) / \ln \left(h_{i-1} / h_{i}\right)$ with $e_{i}:=\left\|u-u_{h_{i}}\right\|_{d G}$, coincide with the theoretically predicted rates of convergence from Theorem 3.5 for smooth solutions $u$; see also Remark 3.6. To be more precise, we observe that $\left\|u-u_{h}\right\|_{d G}$ behaves like $O\left(h^{p}\right)$, where $p$ is the B-spline degree.

${ }^{1} \mathrm{G}+\mathrm{SMO}=$ Geometry plus Simulation MOdules; see also http: / / gs . jku . at / gi smo. 


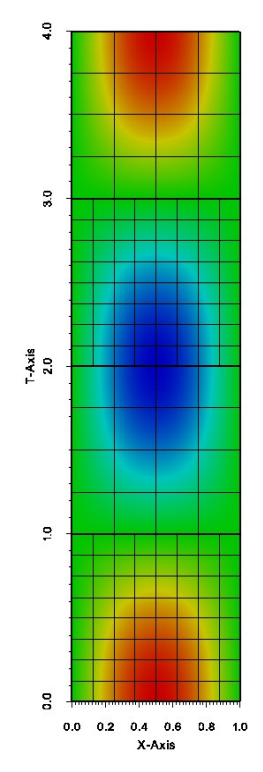

(a)

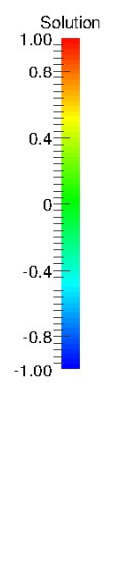

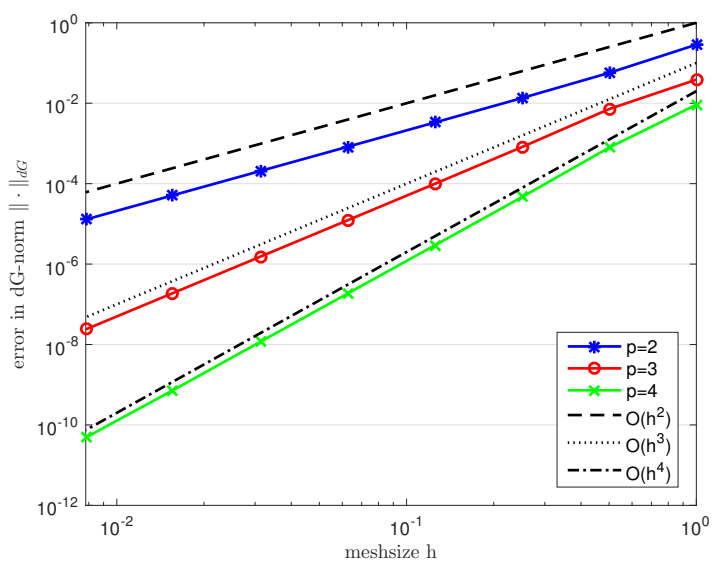

(b)

FIG. 5.1. (a) The solution $u_{h}$ on $Q$ having non-matching meshes across the interface after two uniform refinements of the initial mesh. (b) Convergence plots for polynomial degrees $p=\{2,3,4\}$.

5.2. Parallel solver studies. Here we apply the parallel multigrid solver that was introduced in Section 4 to solve the arising linear systems (4.1) for the case $p=1$, i.e., for lowest-order splines. In detail, we consider the simulation time $T=1$ and the computational domain $\Omega \subset \mathbb{R}^{3}$ given by the control points $(0,0,0)^{\top},(1,0,0)^{\top},(1,1,0)^{\top},(0,1,0)^{\top}$, $(-1 / 4,-1 / 4,1)^{\top},(1,0,1)^{\top},(1,1,1)^{\top},(-1 / 4,5 / 4,1)^{\top}$; see also Figure 5.2. For the initial space-time mesh, we use one space-time patch $(N=1)$ which is decomposed into 64 elements in space and 8 elements with respect to time. We then apply uniform refinement with respect to space, and, at the same time, we increase the number of space-time patches by a factor of two, i.e., we apply uniform refinement in space and time. Throughout all computations, we use the parameter $\theta_{n}=0.2$ for all space-time patches. Moreover, we assemble the linear systems and apply the parallel space-time multigrid solver, discussed in Section 4, as a preconditioner for the GMRES method. For the problem in space, we make use of the software library MFEM ${ }^{2}$, where the AMG library hypre is used as parallel solver in space. For the time parallelization, we use the software developed in [20]. For all examples, we stop the GMRES method when a relative residual error of $10^{-12}$ is reached.

In Table 5.2, we present the numerical results for the manufactured solution

$$
u(x, t)=\sin \left(\pi x_{1}\right) \sin \left(\pi x_{2}\right) \sin \left(\pi x_{3}\right) \sin (\pi t),
$$

which is regular. For this example, we observe the optimal convergence rates in the dG-norm, which are predicted by the theory given in Theorem 3.5. Furthermore, we also obtain quite small iteration numbers for the preconditioned GMRES method. In Table 5.2, we denote the number of cores which are used for the hypre AMG solver and the number of cores which are used for the time parallelization by $c_{x}$ and $c_{t}$, respectively. Hence, we use $c_{x} c_{t}$ cores overall. To fit the problem into the memory of the machine, we increase the number of cores in space

\footnotetext{
${ }^{2}$ MFEM = Modular Finite Element Methods; see also mfem . org
} 


\section{ETNA}

Kent State University and

Johann Radon Institute (RICAM)
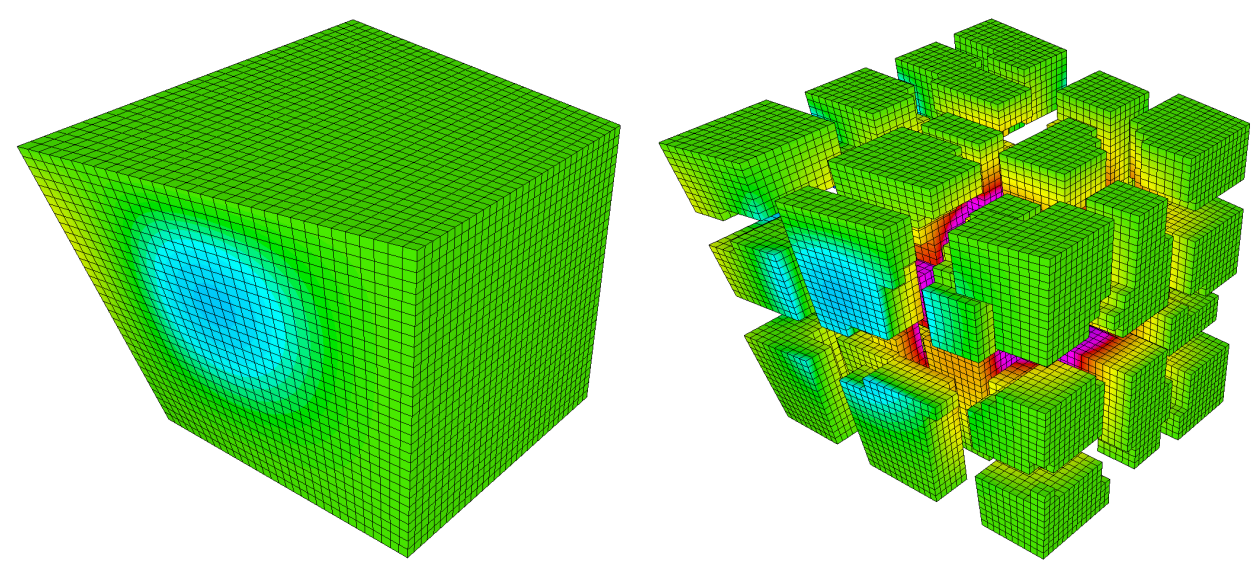

FIG. 5.2. Computational spatial domain $\Omega$ decomposed into 4096 elements (left) and distributed over 32 processors (right). The numerical solution given in Table 5.2 is plotted at $t=0.5$.

TABLE 5.2

Convergence results in $d G$-norm for a regular solution as well as iteration numbers and solution times for the parallel space-time multigrid preconditioned GMRES method.

\begin{tabular}{c|r|rc|rr|r|r|r}
$N$ & overall dof & $\left\|u-u_{h}\right\|_{d G}$ & eoc & $c_{x}$ & $c_{t}$ & cores & iter & time [s] \\
\hline 1 & 1125 & $3.56223 \mathrm{E}-01$ & - & 1 & 1 & 1 & 1 & 0.03 \\
2 & 13122 & $1.77477 \mathrm{E}-01$ & 1.01 & 1 & 2 & 2 & 13 & 1.87 \\
4 & 176868 & $8.86255 \mathrm{E}-02$ & 1.00 & 1 & 4 & 4 & 15 & 21.47 \\
8 & 2587464 & $4.42868 \mathrm{E}-02$ & 1.00 & 4 & 8 & 32 & 15 & 100.48 \\
16 & 39546000 & $2.21376 \mathrm{E}-02$ & 1.00 & 32 & 16 & 512 & 17 & 94.32 \\
32 & 618246432 & $1.10675 \mathrm{E}-02$ & 1.00 & 256 & 32 & 8192 & 17 & 162.90 \\
64 & 9777365568 & $5.53340 \mathrm{E}-03$ & 1.00 & 2048 & 64 & 131072 & 17 & 211.33
\end{tabular}

and time for $N>8$. In detail, we double the number of cores with respect to time, and we increase the number of cores with respect to space by a factor of 8 for each level. This results in an almost constant workload for each processor for $N \geq 8$. In Table 5.2, we observe that the solution times increase for $N=16$ and $N=32$. This does not lead to a completely perfect weak scaling behavior. This is mainly affected by the efficiency of the parallel AMG solver hypre. In particular, one iteration of the AMG solver needs 2.20, 2.01, 2.71, 3.54 seconds for $N=8,16,32,64$, respectively, which explains the computational times given in Table 5.2. Finally, we can solve a linear system consisting of 9777365568 unknowns in less than 4 minutes.

In Table 5.3, we give the convergence rates for the manufactured solution

$$
u(x, t)=\cos \left(\beta x_{1}\right) \cos \left(\beta x_{2}\right) \cos \left(\beta x_{3}\right)(1-t)^{\alpha} \quad \in H^{s, \alpha+\frac{1}{2}-\varepsilon}(Q)
$$

for $\alpha=0.75, \beta=0.3$, an arbitrary $s \geq 2$, and an arbitrary small $\varepsilon>0$. This solution has lower regularity with respect to time; see also [40]. By (3.12) in Theorem 3.5, the asymptotic convergence rate with respect to $h$ is then (almost) given by 0.75 . In Table 5.3, we observe the expected reduced convergence rates predicted by Theorem 3.5. We also observe that the solver is not effected at all by the regularity of the solution.

All parallel computations have been performed on the cluster Vulcan BlueGene/Q at Livermore, USA. 


\section{ETNA}

Kent State University and

Johann Radon Institute (RICAM)

TABLE 5.3

Convergence results in $d G$-norm for a low regularity solution as well as iteration numbers and solution times for the parallel space-time multigrid preconditioned GMRES method.

\begin{tabular}{c|r|cc|rr|r|r|r}
$N$ & overall dof & $\left\|u-u_{h}\right\|_{d G}$ & eoc & $c_{x}$ & $c_{t}$ & cores & iter & time [s] \\
\hline 1 & 1125 & $1.58022 \mathrm{E}-02$ & - & 1 & 1 & 1 & 1 & 0.03 \\
2 & 13122 & $8.88627 \mathrm{E}-03$ & 0.83 & 1 & 2 & 2 & 13 & 2.00 \\
4 & 176868 & $5.41668 \mathrm{E}-03$ & 0.71 & 1 & 4 & 4 & 15 & 21.48 \\
8 & 2587464 & $3.33881 \mathrm{E}-03$ & 0.70 & 4 & 8 & 32 & 15 & 100.57 \\
16 & 39546000 & $2.05545 \mathrm{E}-03$ & 0.70 & 32 & 16 & 512 & 17 & 94.43 \\
32 & 618246432 & $1.25859 \mathrm{E}-03$ & 0.71 & 256 & 32 & 8192 & 17 & 171.83 \\
64 & 9777365568 & $7.65921 \mathrm{E}-04$ & 0.72 & 2048 & 64 & 131072 & 17 & 211.49
\end{tabular}

6. Conclusions. We have presented and analyzed a time-multipatch discontinuous Galerkin space-time IgA method for solving initial-boundary value problems for linear parabolic partial differential equations. The method proposed uses discontinuous Galerkin techniques with time-upwind fluxes for establishing the communication of the discrete solution across the time-patch interfaces. Furthermore, time-upwind diffusion techniques were used for stabilizing the time discretization within each patch. A complete discretization error analysis was developed in a suitable energy norm including the case where the solution can exhibit different regularity behavior with respect to the space and time directions. The convergence rate estimates were confirmed by numerical experiments. We have proposed fast techniques for generating and solving the huge system of IgA equations on massively parallel computers. The parallel experiments were performed for a 3D spatial domain $\Omega$ yielding a $4 \mathrm{D}$ space-time cylinder $Q=\Omega \times(0, T)$ but only for the case $p=1$, where the IgA coincides with the FEM. In this paper, we have always assumed that the spatial computational domain $\Omega$ has a singlepatch representation. The multipatch representation of $\Omega$, which is more important in practice, in connection with dG coupling in space [36] and Dual-Primal IsogEometric Tearing and Interconnection (IETI-DP) solution techniques [22, 23] is work in progress. This approach is quite flexible with respect to the adaption of the discretization to the behavior of the solution. At the same time, it allows a fast generation and solution of the system of IgA equations due to the fact that the local space-time patches into which the space-time cylinder $Q$ is decomposed have tensor-product structure. A complete unstructured decomposition of the space-time cylinder $Q$ into patches, which was considered in [39], loses this structure, and, therefore, an efficient implementation is cumbersome.

Acknowledgments. The first author would like to thank the Austrian Science Fund (FWF) for the support through the DK W1214-04, while the second author and the last author were supported by the FWF grant NFN S117-03. Moreover we want to thank P. Vassilevski for the possibility to compute on the Vulcan Cluster in Livermore. Furthermore, the authors would like to express their thanks to the anonymous referees for their helpful hints and suggestions.

\section{REFERENCES}

[1] R. ANDREev, Stability of sparse space-time finite element discretizations of linear parabolic evolution equations, IMA J. Numer. Anal., 33 (2013), pp. 242-260.

[2] R. ANDreev, O. SCHERZER, AND W. ZULEHNER, Simultaneous optical flow and source estimation: spacetime discretization and preconditioning, Appl. Numer. Math., 96 (2015), pp. 72-81.

[3] I. BABUŠKA AND T. JANIK, The $h$-p version of the finite element method for parabolic equations. Part I. The p-version in time, Numer. Methods for Partial Differential Equations, 5 (1989), pp. 363-399.

[4] - The $h-p$ version of the finite element method for parabolic equations. II. The h-p version in time, Numer. Methods Partial Differential Equations, 6 (1990), pp. 343-369. 


\section{ETNA}

Kent State University and

Johann Radon Institute (RICAM)

[5] R. E. BAnK AND M. S. MetTI, An error analysis of some higher order space-time moving finite elements, Comput. Vis. Sci., 16 (2013), pp. 219-229.

[6] R. E. Bank, P. S. VAssilevski, AND L. T. Zikatanov, Arbitrary dimension convection-diffusion schemes for space-time discretizations, J. Comput. Appl. Math., 310 (2017), pp. 19-31.

[7] Y. BAZilevs, L. Beirão da Veiga, J. A. Cottrell, T. J. R. Hughes, and G. Sangalli, Isogeometric analysis: approximation, stability and error estimates for h-refined meshes, Math. Models Methods Appl. Sci., 16 (2006), pp. 1031-1090.

[8] M. BEHR, Simplex space-time meshes in finite element simulations, Internat. J. Numer. Methods Fluids, 57 (2008), pp. 1421-1434.

[9] L. Beirao DA Veiga, A. Buffa, J. Rivas, ANd G. SANGALli, Some estimates for $h$ - $p$ - $k$ refinement in isogeometric analysis, Numer. Math., 118 (2011), pp. 271-305.

[10] L. Beirão DA Veiga, A. Buffa, G. SANGALli, AND R. VÁZquez, Mathematical analysis of variational isogeometric methods, Acta Numer., 23 (2014), pp. 157-287.

[11] L. BeIRÃo DA VeIGA, D. Cho, AND G. SANGALLI, Anisotropic NURBS approximation in isogeometric analysis, Comput. Methods Appl. Mech. Engrg., 209/212 (2012), pp. 1-11.

[12] D. BraEss, Finite Elements: Theory, Fast Solvers, and Applications in Elasticity Theory, 3rd. ed., Cambridge University Press, Cambridge 2007.

[13] A. N. BRooks AND T. J. R. Hughes, Streamline upwind/Petrov-Galerkin formulations for convection dominated flows with particular emphasis on the incompressible Navier-Stokes equations, Comput. Methods Appl. Mech. Engrg., 32 (1982), pp. 199-259.

[14] J. A. Cottrell, T. Hughes, AND Y. BAZILEvs, Isogeometric Analysis, Toward Integration of CAD and FEA, Wiley, Chichester, 2009.

[15] C. DE Boor, A Practical Guide to Splines, rev. ed., Springer, New York, 2001.

[16] J. A. EVANS AND T. J. R. HUGHES, Explicit trace inequalities for isogeometric analysis and parametric hexahedral finite elements, Numer. Math., 123 (2013), pp. 259-290.

[17] R. D. FAlgout, J. E. JONES, AND U. Meier YANG, The design and implementation of hypre, a library of parallel high performance preconditioners, in Numerical Solution of Partial Differential equations on Parallel Computers, A. M. Bruaset and A. Tveito, eds., vol. 51 of Lect. Notes Comput. Sci. Eng., Springer, Berlin, 2006, pp. 267-294.

[18] R. D. FAlgout AND U. MEIER YANG, hypre: a library of high performance preconditioners, Proceedings of the International Conference on Computational Science-Part III, P. M. A. Sloot, C. J. K. Tan, J. J. Dongarra, and, A. G. Hoekstra, eds., Springer, London, 2002, pp. 632-641.

[19] M. J. GANDER, 50 years of time parallel time integration, in Multiple Shooting and Time Domain Decomposition Methods, T. Carraro, M. Geiger, S. Körkel, and R. Rannachev, eds., vol. 9 of Contrib. Math. Comput. Sci., Springer, Cham, 2015, pp. 69-113.

[20] M. J. GANDER AND M. NEUMÜLLER, Analysis of a new space-time parallel multigrid algorithm for parabolic problems, SIAM J. Sci. Comput., 38 (2016), pp. A2173-A2208.

[21] P. HANSBO, Space-time oriented streamline diffusion methods for nonlinear conservation laws in one dimension, Comm. Numer. Methods Engrg., 10 (1994), pp. 203-215.

[22] C. Hofer, Parallelization of continuous and discontinuous Galerkin dual-primal isogeometric tearing and interconnecting methods, Comput. Math. Appl., 74 (2017), pp. 1607-1625.

[23] C. HOFER AND U. LANGER, Dual-primal isogeometric tearing and interconnecting solvers for multipatch dG-IgA equations, Comput. Methods Appl. Mech. Engrg., 316 (2017), pp. 2-21.

[24] K. HöLLIG, Finite Element Methods with B-Splines, SIAM, Philadelphia, 2003.

[25] T. J. R. Hughes, J. A. CotTRELl, AND Y. BAZILEVs, Isogeometric analysis: CAD, finite elements, NURBS, exact geometry and mesh refinement, Comput. Methods Appl. Mech. Engrg., 194 (2005), pp. 4135-4195.

[26] C. Johnson, U. NÄVERT, AND J. PITKÄRANTA, Finite element methods for linear hyperbolic problems, Comput. Methods Appl. Mech. Engrg., 45 (1984), pp. 285-312.

[27] C. Johnson AND J. SARANEn, Streamline diffusion methods for the incompressible Euler and Navier-Stokes equations, Math. Comp., 47 (1986), pp. 1-18.

[28] E. Karabelas, Space-time Discontinuous Galerkin Methods for Cardic Electro-Mechanics, PhD. Thesis, Institute of Computational Mathematics, Technische Universität Graz, Graz, 2015.

[29] E. Karabelas AND M. NeUmÜLlER, Generating admissible space-time meshes for moving domains in $(d+1)$-dimensions, Tech. Report, NuMa-Report 2015-07, Institute for Computational Mathematics Johannes Kepler University Linz, Linz, 2015.

[30] O. A. Ladyzhenskaya, The Boundary Value Problems of Mathematical Physics, Springer, New York, 1985.

[31] O. A. Ladyzenskaja, V. A. SOlonniKov, And N. N. URALT'SEVA, Linear and Quasilinear Equations of Parabolic Type, Nauca, Moscow, 1967. Translated in AMS, Providence, 1968.

[32] J. LANG, Adaptive Multilevel Solution of Nonlinear Parabolic PDE Systems. Theory, Algorithm, and Applications, Springer, Berlin, 2001.

[33] U. LANGer, S. MATCUleVich, And S. RePin, Functional type error control for stabilised space-time IgA approximations to parabolic problems, in Large-Scale Scientific Computing (LSSC 2017), I. Lirkov and 
S. Margenov, eds., vol. 10665 of Lecture Notes in Computer Science (LNCS), Springer, Cham, 2017, pp. 55-65.

[34] U. Langer, S. Moore, And M. Neumüller, Space-time isogeometric analysis of parabolic evolution equations, Comput. Methods Appl. Mech. Engrg., 306 (2016), pp. 342-363.

[35] U. Langer, M. Neumüller, AND I. Toulopoulos, Multipatch space-time isogeometric analysis of parabolic diffusion problems, in Large-Scale Scientific Computing (LSSC 2017), I. Lirkov and S. Margenov, eds., vol. 10665 of Lecture Notes in Computer Science (LNCS), Springer, Cham, 2017, pp. 21-32.

[36] U. LANGER AND I. TOULOPOUlOS, Analysis of multipatch discontinuous Galerkin IgA approximations to elliptic boundary value problems, Comput. Vis. Sci., 17 (2015), pp. 217-233.

[37] S. LARSSON AND M. MOLTENI, Numerical solution of parabolic problems based on a weak space-time formulation, Comput. Methods Appl. Math., 17 (2017), pp. 65-84.

[38] C. Mollet, Stability of Petrov-Galerkin discretizations: application to the space-time weak formulation for parabolic evolution problems, Comput. Methods Appl. Math., 14 (2014), pp. 231-255.

[39] S. Moore, Nonstandard Discretization Strategies In Isogeometric Analysis for Partial Differential Equations, $\mathrm{PhD}$. Thesis, Johann Radon Institute for Computational and Applied Mathematics, Austrian Academy of Sciences, University Linz, Linz, 2017.

[40] M. NeumÜLLER, Space-Time Methods: Fast Solvers and Applications, vol. 20 of Monographic Series TU Graz, TU Graz, Graz 2013.

[41] M. NeumÜLler And O. STEInbach, Refinement of flexible space-time finite element meshes and discontinuous Galerkin methods, Comput. Vis. Sci., 14 (2011), pp. 189-205.

[42] C. Petra, O. Schenk, AND M. Anitescu, Real-time stochastic optimization of complex energy systems on high-performance computers, IEEE Comput. Sci. \& Engrg., 16 (2014), pp. 32-42.

[43] C. G. Petra, O. Schenk, M. Lubin, And K. GÄertner, An augmented incomplete factorization approach for computing the Schur complement in stochastic optimization, SIAM J. Sci. Comput., 36 (2014), pp. C139-C162.

[44] L. PIEGL AND W. TILlER, The NURBS Book, Springer, Berlin, 1997.

[45] L. L. Schumaker, Spline Functions: Basic Theory, 3rd ed., Cambridge University Press, Cambridge, 2007.

[46] C. SCHWAB AND R. STEVENSON, Space-time adaptive wavelet methods for parabolic evolution problems, Math. Comp., 78 (2009), pp. 1293-1318.

[47] I. SMEARS, Robust and efficient preconditioners for the discontinuous Galerkin time-stepping method, IMA J. Numer. Anal., 37 (2017), pp. 1961-1985.

[48] O. STEINBACH, Space-time finite element methods for parabolic problems, Comput. Methods Appl. Math., 15 (2015), pp. 551-566.

[49] M. STYNES, Steady-state convection-diffusion problems, Acta Numer., 14 (2005), pp. 445-508.

[50] K. Takizawa, B. Henicke, A. Puntel, N. Kostov, and T. TeZduyar, Space-time techniques for computational aerodynamics modeling of flapping wings of an actual locust, Comput. Mech., 50 (2012), pp. 743-760.

[51] K. Takizawa, N. Kostov, A. Puntel, B. Henicke, and T. Tezduyar, Space-time computational analysis of bio-inspired flapping-wing aerodynamics of a micro aerial vehicle, Comput. Mech., 50 (2012), pp. 761-778.

[52] K. TAKIZAWA AND T. E. TEZDUYAR, Multiscale space-time fluid-structure interaction techniques, Comput. Mech., 48 (2011), pp. 247-267.

[53] - Space-time computation techniques with continuous representation in time (ST-C), Comput. Mech., 53 (2014), pp. 91-99.

[54] K. Takizawa, T. E. Tezduyar, S. Mcintyre, N. Kostov, R. Kolesar, and C. Habluetzel, Space-time VMS computation of wind-turbine rotor and tower aerodynamics, Comput. Mech., 53 (2014), pp. $1-15$.

[55] V. THOMÉE, Galerkin Finite Element Methods for Parabolic Problems, 2nd. ed., Springer, Berlin, 2006.

[56] I. TOULOPOULOS, Bubble stabilized space-time finite element methods of parabolic evolution problems, Tech. Report, RICAM Report 2017-19, Johann Radon Institute for Computational and Applied Mathematics, Austrian Academy of Sciences, Linz, 2017.

[57] K. URBAN AND A. T. PATERA, An improved error bound for reduced basis approximation of linear parabolic problems, Math. Comp., 83 (2014), pp. 1599-1615.

[58] J. J. W. VAN DER VEGT AND S. RHEBERGEN, hp-multigrid as smoother algorithm for higher order discontinuous Galerkin discretizations of advection dominated flows. Part I. Multilevel analysis., J. Comput. Phys., 231 (2012), pp. 7537-7563.

[59] _ - hp-multigrid as smoother algorithm for higher order discontinuous Galerkin discretizations of advection dominated flows. Part II. Optimization of the Runge-Kutta smoother, J. Comput. Phys., 231 (2012), pp. 7564-7583. 
ETNA

Kent State University and

Johann Radon Institute (RICAM)

[60] J. J. W. VAN DER VEGT AND H. VAN DER VEN, Space-time discontinuous Galerkin finite element method with dynamic grid motion for inviscid compressible flows. I. General formulation, J. Comput. Phys., 182 (2002), pp. 546-585. 